Ninth Mexican School on Particles and Fields 9-19 August 2000, Metepec, Puebla, Mexico

\title{
Disoriented Chiral Condensates in High-Energy Nuclear Collisions
}

\author{
Jørgen Randrup \\ Lawrence Berkeley National Laboratory, Berkeley, California 94720, USA
}

\begin{abstract}
This brief lecture series discusses how our current understanding of chiral symmetry may be tested more globally in high-energy nuclear collisions by suitable extraction of pionic observables. After briefly recalling the general features of chiral symmetry, we focus on the $\mathrm{SU}(2)$ linear $\sigma$ model and show how a semi-classical meanfield treatment makes it possible to calculate its statistical properties, including the chiral phase diagram. Subsequently, we consider scenarios of relevance to high-energy collisions and discuss the features of the ensuing non-equilibrium dynamics and the associated characteristic signals. Finally, we illustrate how the presence of vacuum fluctuations or the inclusion of strangenesss may affect the results quantitatively.
\end{abstract}

\section{INTRODUCTION}

The advent of ever more powerful heavy-ion accelerators has made it possible to study strongly interacting matter over a wide range of physical conditions. In particular, at the unprecedented energy densities now within reach at RHIC two fundamental phase changes are expected to occur. One is the dissolution of ordinary hadrons into a deconfined quark-gluon plasma phase, which has long been a principal research objective. The other, on which the present lectures are focussed, is the approximate restoration of chiral symmetry. This topic has gained increasing interest in recent years because of the recognition that the rapid non-equilibrium dynamics may produce coherent oscillations of the pion field with observable consequences. Reviews of this phenomenon, commonly referred to as disoriented chiral condensates (DCC), are given in Refs. [1-3].

Since the $u$ and $d$ quark masses are fairly small, the basic QCD Lagrangian is approximately invariant with respect to chiral transformations and it is therefore expected that matter at high energy density will display approximate chiral symmetry. However, due to the self-interaction of the fields, this symmetry is spontaneously broken at lower energy. In particular, the ordinary vaccum exhibits a finite value of the order parameter, $\langle q \bar{q}\rangle=f_{\pi}$, and the associated pionic excitations are approximately massless on the hadronic scale. A pedagogical introduction to the basic concepts of chiral symmetry in nuclear physics is given in Ref. [4]. 
The most popular theoretical tool for studies of DCC phenomena has been the linear $\sigma$ model [5] which describes the $\mathrm{O}(4)$ chiral field $\boldsymbol{\phi}=(\sigma, \boldsymbol{\pi})$ by means of a simple effective quartic interaction (see Refs. [6-14] for some examples),

$$
\mathcal{L}=\frac{1}{2} \partial_{\mu} \phi \circ \partial^{\mu} \boldsymbol{\phi}-\frac{\lambda}{4}\left(\phi^{2}-v^{2}\right)^{2}+H \sigma \quad \Rightarrow \quad\left[\square+\lambda\left(\phi^{2}-v^{2}\right)\right] \boldsymbol{\phi}=H \hat{\sigma} .
$$

The three model parameters, $\lambda, v$, and $H$, can be fixed by specifying the pion decay constant, $f_{\pi}=92 \mathrm{MeV}$, the free pion mass, $m_{\pi}=138 \mathrm{MeV}$, and the mass of the nominal $\sigma$ meson (which is rather uncertain), $m_{\sigma}=600 \mathrm{MeV}$. In vacuum, the chiral field is aligned with the $\sigma$ direction, $\boldsymbol{\phi}_{\mathrm{vac}}=\left(f_{\pi}, \mathbf{0}\right)$, and at low temperatures the agitations of the field represent nearly free $\sigma$ and $\pi$ mesons. This simplest version of the model is appropriate only in a baryon-free environment, such as the central rapidity region at RHIC, and it needs to be appropriately extended when baryons are present (for an example of this, see Ref. [8]).

Within this conceptually simple framework, the basic mechanism of DCC formation is readily depicted, as illustrated in Fig. 1: The early violent part of a collision event produces an extended region of space within which the energy density is so high that chiral symmetry is temporarily nearly restored. As the collision evolves further, the energy density drops so rapidly that the chiral degrees of freedom fall out of equilibrium, leading to large-amplitude long-wavelength isospin-directed oscillations of the pion field. This unique phenomenon would have a number of specific observational consequences, including anomalous multiplicity distributions of the soft pions and a significant enhancement of dilepton production.

This lecture series reviews efforts to address the phenomenon quantitatively within the framework of the linear $\sigma$ model.

\section{STATISTICAL EQUILIBRIUM}

In order to understand the key features of DCC formation, which is inherently a non-equilibrium phenomenon, it is useful to know the equilibrium properties of the system. In this section we discuss how statistical equilibrium may be addressed quantitatively within a semi-classical framework that is readily extendable to arbitrary time-dependent scenarios. We follow Ref. [15] where more details are given.

\section{Mean-Field Treatment of the Linear $\sigma$ Model}

The present considerations are restricted to systems confined within a cubic box of volume $\Omega=L^{3}$; the continuum limit is then approached (rapidly) as $L \rightarrow \infty$. A given microscopic state of the system is characterized by the field $\phi(\boldsymbol{r})$ and its time derivative $\boldsymbol{\psi}(\boldsymbol{r})$, both of which are real functions and have periodic boundary conditions. (The separate notation for the time derivative of the field emphasizes that it represents independent information; it is also notationally advantageous.) 


\section{Idealized illustration of DCC formation:}

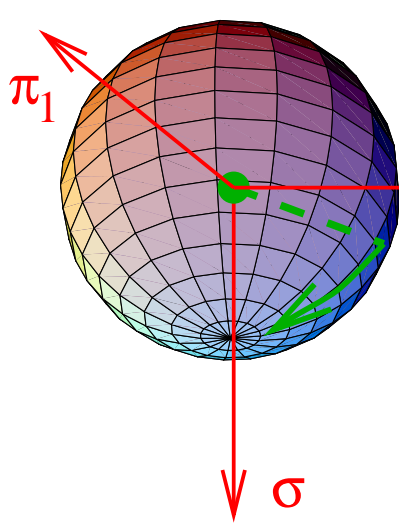

The system starts at high temperature where chiral symmetry is nearly restored:

\section{$\pi_{2}$}

Rapid expansion causes a quench that renders the symmetric state unstable:

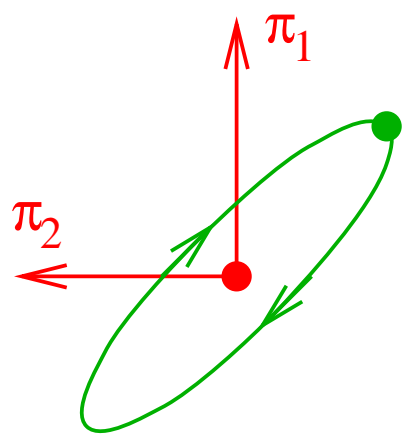

The order parameter quickly grows in some chiral direction, breaking the chiral symmetry:

The later relaxation displays large-amplitude isospin-directed oscillations of the pion field:
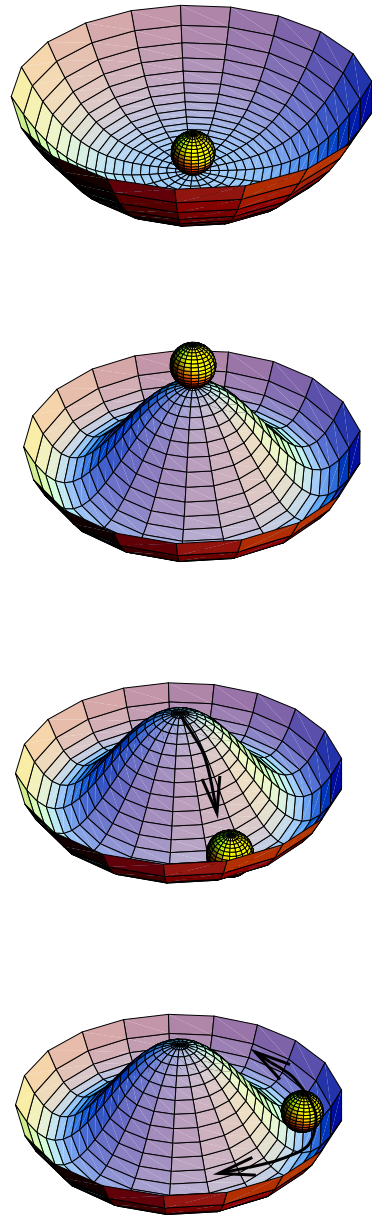

FIGURE 1. The mechanism leading to DCC formation is illustrated for the simplified worlds in which the isospin space has only two (left-hand side) or one (right-hand side) dimension. In the originally conceived DCC quench scenario, the system is initially so highly agitated that the $\mathrm{O}(4)$ order parameter $(\sigma, \pi)$ is situated at the single minimum of the effective potential near the origin. The system then expands and/or cools so rapidly that the effective potential quickly reverts to its low-temperature form, in which a nearly degenerate minimum appears as a trough on the surface of the 4 -hypersphere with radius $f_{\pi}, \sigma^{2}+\pi^{2} \approx f_{\pi}^{2}$. Since the early rapid growth of the order parameter is primarily in the radial direction, the generated vector current $\boldsymbol{\pi} \times \dot{\pi}$ is very small and the subsequent oscillatory relaxation towards the ground state is therefore well directed in isospace (lower left illustration). The emitted pion radiation is then correspondingly isospin polarized, resulting in a very broad distribution of the neutral fraction $f=N_{0} /\left(N_{-}+N_{0}+N_{+}\right)$. 
It is useful to separate the field into two parts, $\phi(\boldsymbol{r})=\boldsymbol{\phi}+\boldsymbol{\delta} \phi(\boldsymbol{r})$. The spatial average of the field, $\phi=\langle\phi(\boldsymbol{r})\rangle$ is identified with the $\overline{\mathrm{O}}(4)$ order parameter associated with that particular field configuration. The components of the field fluctuation $\boldsymbol{\delta} \phi(\boldsymbol{r})$ along the $\mathrm{O}(4)$ direction of the order parameter is denoted by $\delta \phi_{\|}(\boldsymbol{r})$ and $\boldsymbol{\delta} \phi_{\perp}(\boldsymbol{r})$ is its component perpendicular to $\boldsymbol{\phi}$. A similar decomposition can be made for the time derivative, $\boldsymbol{\psi}(\boldsymbol{r})=\boldsymbol{\psi}+\boldsymbol{\delta} \boldsymbol{\psi}(\boldsymbol{r})$. This separation makes it possible to interpret the field fluctuations as quasiparticle excitations relative to the environment (or "effective vacuum") characterized by the temperature $T$ and the magnitude of order parameter, $\phi_{0}$.

Moreover, the separation of the field invites the application of the Hartree factorization technique. The first step, taking the spatial average of the full equation of motion (1), leads to an equation of motion for the order parameter $\underline{\phi}[16]$,

$$
\left[\partial_{t}^{2}+\mu_{0}^{2}\right] \underline{\boldsymbol{\phi}}=H \hat{\boldsymbol{e}}_{\sigma}
$$

The associated effective mass $\mu_{0}$ for the order parameter is given by

$$
\mu_{0}^{2}=\lambda\left[\phi_{0}^{2}+\prec \delta \phi^{2} \succ+2 \prec \delta \phi_{\|}^{2} \succ-v^{2}\right] .
$$

Here $\phi_{0} \equiv|\underline{\phi}|$ is the magnitude of the order parameter. Moreover, $\prec \delta \phi_{\|}^{2} \succ$ is the thermal average of the field fluctuation along $\underline{\phi}$, while $\prec \delta \phi^{2} \succ=\prec \delta \phi_{\|}^{2} \succ+3 \prec$ $\delta \phi_{\perp}^{2} \succ$ is the total field fluctuation (the three perpendicular fluctuation components have the same thermal average and $\prec \delta \phi_{\perp}^{2} \succ$ denotes just one of them). Terms vanishing in thermal equilibrium have been ignored, namely correlations between field fluctuations in different $O(4)$ directions, $\prec \delta \phi_{\|} \delta \phi_{\perp} \succ=\mathbf{0}$, and averages of odd powers of field fluctuations, $\prec \delta \phi^{2} \boldsymbol{\delta} \phi \succ=\mathbf{0}$.

The above result can be used to determine the phase diagram, since the order parameter experiences no forces in equilibrium, $\partial_{t}^{2} \underline{\phi}=\mathbf{0}$. Thus, at a given temperature $T$, the stationary points of $\underline{\phi}$ are determined by $\mu_{0}^{2} \boldsymbol{\phi}=H \hat{\boldsymbol{e}}_{\sigma}$, which implies that the equilibria are located on the $\sigma$ axis. If $\chi_{0}$ is the angle between $\underline{\phi}$ and the $\sigma$ direction, the aligned component of the order parameter is given by $\sigma_{0}=\phi_{0} \cos \chi_{0}$ and the equilibrium condition then amounts to the relation $\mu_{0}^{2} \sigma_{0}=H$. When the free pion mass vanishes, so does $H$. The condition for obtaining an equilibrium for a finite magnitude of the order parameter, $\phi_{0}>0$ (in which chiral symmetry is thus spontaneously broken), then amounts to the requirement that $\mu_{0}$ be zero. This feature is a manifestation of the Goldstone theorem [17].

In order to calculate the field fluctuations entering in the expression (3) for $\mu_{0}^{2}$, it is necessary to know the quasiparticle masses. Subtracting the above equation of motion (2) from the complete equation (1) and applying a suitable Hartree-type factorization, approximate Klein-Gordon equations of motion can be obtained for the field fluctuations $\boldsymbol{\delta} \phi=\left(\delta \phi_{\|}, \boldsymbol{\delta} \phi_{\perp}\right)[15]$,

$$
\left[\square+\mu_{\|}^{2}\right] \delta \phi_{\|}=0, \quad\left[\square+\mu_{\perp}^{2}\right] \delta \phi_{\perp}=\mathbf{0} .
$$

The effective quasi-particle masses $\mu_{\|}$and $\mu_{\perp}$ are determined by the gap equations, 


$$
\begin{aligned}
& \mu_{\|}^{2}=\lambda\left[3 \phi_{0}^{2}+\prec \delta \phi^{2} \succ+2 \prec \delta \phi_{\|}^{2} \succ-v^{2}\right], \\
& \mu_{\perp}^{2}=\lambda\left[\phi_{0}^{2}+\prec \delta \phi^{2} \succ+2 \prec \delta \phi_{\perp}^{2} \succ-v^{2}\right],
\end{aligned}
$$

and the corresponding quasiparticle dispersion relations are $\left(\omega_{k}^{\|}\right)^{2}=k^{2}+\mu_{\|}^{2}$ and $\left(\omega_{k}^{\perp}\right)^{2}=k^{2}+\mu_{\perp}^{2}$. Finally, the thermal fluctuations are given by the usual expressions for bosonic fields,

$$
\begin{aligned}
& \prec \delta \phi_{\|}^{2} \succ=\frac{1}{\Omega} \sum_{\mathbf{k} \neq \mathbf{0}} \frac{1}{\omega_{k}^{\|}} \frac{1}{\mathrm{e}^{\omega_{k}^{\|} / T}-1} \asymp \frac{\mu_{\|} T}{2 \pi^{2}} \sum_{n>0} \frac{1}{n} K_{1}\left(\frac{n \mu_{\|}}{T}\right), \\
& \prec \delta \phi_{\perp}^{2} \succ=\frac{1}{\Omega} \sum_{\mathbf{k} \neq \mathbf{0}} \frac{1}{\omega_{k}^{\perp}} \frac{1}{\mathrm{e}^{\omega_{k}^{\perp} / T}-1} \asymp \frac{\mu_{\perp} T}{2 \pi^{2}} \sum_{n>0} \frac{1}{n} K_{1}\left(\frac{n \mu_{\perp}}{T}\right),
\end{aligned}
$$

where the last relations hold in the continuum limit $(L \rightarrow \infty)$. We note that $\mu_{0}^{2} \leq \mu_{\perp}^{2} \leq \mu_{\|}^{2}$, with the equalities holding for $\phi_{0}=0$. Moreover, the gap equations do not contain the parameter $H$, so the resulting effective masses are independent of the disorientation angle $\chi_{0}$.

Utilizing the expressions (7-8), the coupled equations (5-6) for the effective masses can be solved for specified values of the temperature $T$ and the magnitude of the order parameter, $\phi_{0}$, provided that these quantities are sufficiently large. The critical boundary on a $\phi_{0}-T$ diagram inside which no solution exist, is determined by the vanishing of the transverse mass, $\mu_{\perp}=0$ (see Eq. 9 below).

Figure 2 shows the resulting effective masses $\mu_{\|}$and $\mu_{\perp}$ as functions of $\phi_{0}$, for temperatures $T$ up to well above critical. At any temperature, there is always a physical solution to the coupled equations (5-6) for the effective masses when $\phi_{0} \geq v$. This is easy to see from Eq. (6): At $T=0$, when the fluctuations vanish, we have $\mu_{\perp}^{2}=\lambda\left(\phi_{0}^{2}-v^{2}\right)$ and so $\mu_{\perp}^{2}$ vanishes at $\phi_{0}=v$ and is positive for larger $\phi_{0}$; an increase of $T$ will always increase the fluctuations, and hence the mass. Moreover, we always have $\mu_{\|} \geq \mu_{\perp}$.

Since the field fluctuations and the magnitude of the order parameter contribute to the effective masses in qualitatively similar ways, an increase of the temperature (and thus the fluctuations) will permit a further decrease of $\phi_{0}$, so that the point at which $\mu_{\perp}$ vanishes is moved to ever smaller values of $\phi_{0}$. The appearance of $\mu_{\|}$, considered as a function of $\phi_{0}$, is nearly independent of temperature, except that each curve terminates at the point where the corresponding $\mu_{\perp}$ vanishes. This limiting curve is indicated by the dotted curve on the interval $(0, v)$ and it is elementary to calculate,

$\mu_{\perp}=0: \phi_{0}^{2}=v^{2}-\frac{5}{12} T^{2}-\prec \delta \phi_{\|}^{2} \succ \approx v^{2}-\frac{T^{2}}{12}\left(5+\mathrm{e}^{-\mu_{\|} / T}\right), \mu_{\|}^{2}=\lambda\left(2 v^{2}-T^{2}\right)$.

This behavior continues until the temperature reaches the value $T_{0}$, the lowest temperature for which there is a solution to eqs. (5-6) for all values of $\phi_{0}$. For this particular temperature both effective masses vanish for $\phi_{0}=0$. Consequently, we have $\prec \delta \phi_{j}^{2} \succ=T_{0}^{2} / 12$ and so $T_{0}^{2}=2 v^{2}$, i.e. $T_{0}=122.63 \mathrm{MeV}$ with the adopted 


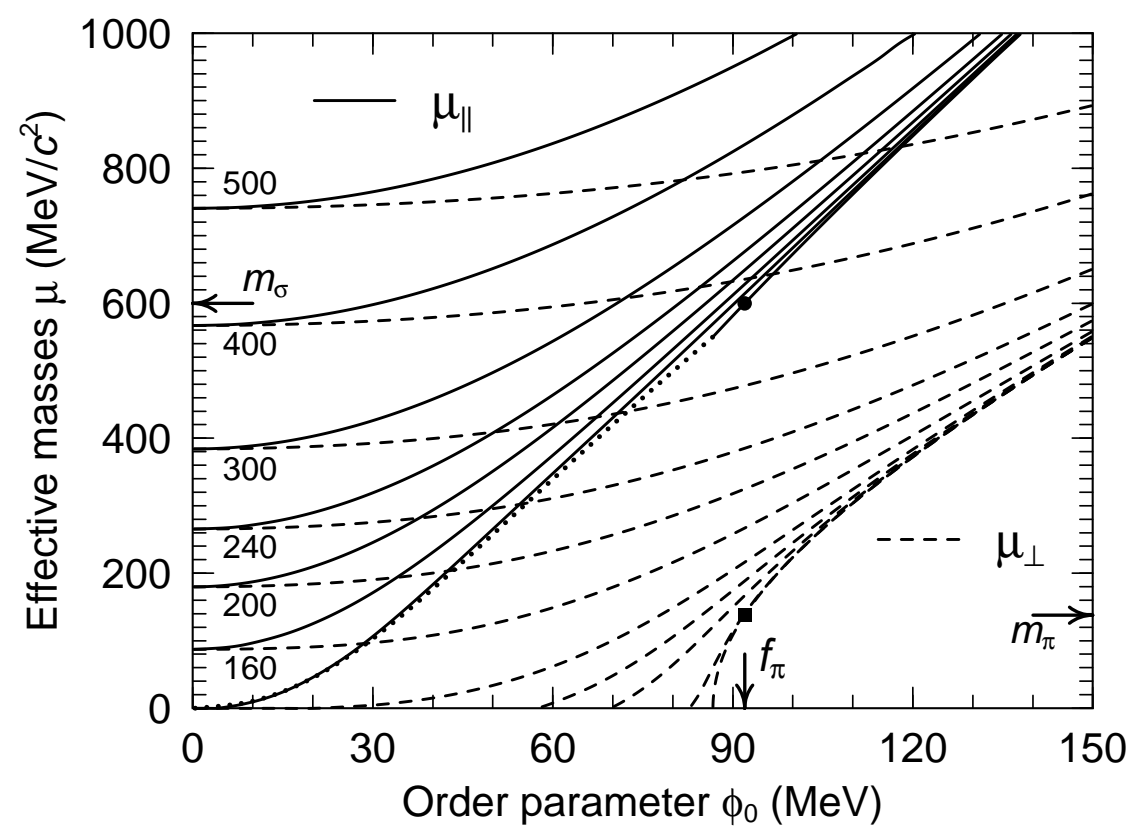

FIGURE 2. Solution to the gap equations. The effective masses $\mu_{\|}$(solid) and $\mu_{\perp}$ (dashed), as functions of the magnitude of the order parameter, $\phi_{0}$, for a range of temperatures: $T=0,40$, $80,100,122.63\left(=T_{0}\right), 160,200,240,300,400,500 \mathrm{MeV}$, calculated in the thermodynamic limit where the box size is large, $L \rightarrow \infty$. For a temperature above $T_{0}$, the two effective-mass curves start out at $\phi_{0}=0$ with degenerate values, whereas below $T_{0}$ they only exist if $\phi_{0}$ is sufficiently large. The corresponding starting points for $\mu_{\|}$are connected by the dotted curve and, since $\mu_{\|}$ is then nearly independent of $T$, only the curve for $T=0$ has been shown. The vertical arrow points to the vacuum value of the order parameter, $\phi_{\mathrm{vac}}=f_{\pi}=92 \mathrm{MeV}$, and the free mass values $\mu_{\|}=m_{\sigma}=600 \mathrm{MeV}$ and $\mu_{\perp}=m_{\pi}=138 \mathrm{MeV}$ are indicated by the horizontal arrows. The locations of the corresponding points in the diagram are shown by the two solid symbols. Since the field fluctuations are rather insensitive to the box size, except near the critical point, the effective masses exhibit only very little size dependence.

parameter values. [ $T_{0}$ is here used to denote that unique value of $T$ for which the effective masses are zero when the order parameter vanishes. It is occasionally referred to as the "critical temperature" and denoted $T_{c}$ but we find this nomenclature unfortunate, since the transition from approximate chiral symmetry to a broken phase generally occurs at significantly higher temperatures, as we shall illustrate later on.] The degeneracy in the masses, $\mu_{\|}=\mu_{\perp}$, is a general consequence of the $O(4)$ rotational symmetry that emerges for $\phi_{0}=0$ and it therefore remains as $T$ is further increased, with the common mass value $\mu_{0}$ increasing steadily. Since the effective mass at $\phi_{0}=0$ is given by $\mu_{0}^{2}=\lambda\left(6 \prec \delta \phi_{j}^{2} \succ-v^{2}\right)$, it becomes proportional to $T$ at high temperatures, $\mu_{0} c^{2} \approx 1.59 T$ for $T \gg v$. 


\section{Partition Function}

The statistical properties of the chiral field are governed by the partition function which, in the semi-classical treatment, is given by

$$
\mathcal{Z}_{T}=\int \mathcal{D}[\phi(\boldsymbol{r}), \boldsymbol{\psi}(\boldsymbol{r})] \mathrm{e}^{-\frac{\Omega}{T} E[\phi(\boldsymbol{r}), \boldsymbol{\psi}(\boldsymbol{r})]}
$$

The functional integral is over all possible field configurations and $E$ is the mean energy density of any such state of the system,

$$
E[\boldsymbol{\phi}(\boldsymbol{r}), \boldsymbol{\psi}(\boldsymbol{r})]=\left\langle\frac{1}{2} \psi^{2}+\frac{1}{2}(\nabla \phi)^{2}+\frac{\lambda}{4}\left(\phi^{2}-v^{2}\right)^{2}-H \sigma\right\rangle=E_{0}+E_{\mathrm{qp}}+\delta V,
$$

with $\psi_{0} \equiv|\underline{\boldsymbol{\psi}}|$. In the last relation, the energy has been decomposed into terms having instructive physical interpretations [15]. The first term in this decomposition is the energy density that would result if all the field fluctuations were put to zero,

$$
E_{0}=\frac{1}{2} \psi_{0}^{2}+\frac{\lambda}{4}\left(\phi_{0}^{2}-v^{2}\right)^{2}-H \phi_{0} \cos \chi_{0}=K_{0}+V_{0} .
$$

It consists of the bare kinetic energy density $K_{0}=\frac{1}{2} \psi_{0}^{2}$ and the bare interaction energy density $V_{0}$.

The second term in $E$ is the energy associated with the gas of independent quasiparticles described by the above Klein-Gordon equations of motion (4),

$$
E_{\mathrm{qp}}=\frac{1}{2}\langle\boldsymbol{\delta} \psi \circ \delta \boldsymbol{\psi}+\nabla \boldsymbol{\delta} \phi \circ \nabla \boldsymbol{\delta} \phi+\delta \phi \circ \boldsymbol{M} \circ \boldsymbol{\delta} \phi\rangle
$$

where $\boldsymbol{M}$ is the $\mathrm{O}(4)$ quasiparticle mass tensor. In thermal equilibrium its major principal axis is oriented along the order parameter, the corresponding eigenvalue is $\mu_{\|}^{2}$, and the other three eigenvalues are equal to $\mu_{\perp}^{2}$.

The last term in $E$ corrects for the fact that the interaction is non-linear,

$$
\begin{aligned}
\delta V & =\frac{\lambda}{4}\left\langle\delta \phi^{4}\right\rangle-\frac{\lambda}{2}\left\langle\delta \phi^{4}\right\rangle_{G} \approx-\frac{\lambda}{4}\left\langle\delta \phi^{4}\right\rangle_{G}=\left\langle\delta \phi^{2}\right\rangle^{2}+2 \operatorname{Tr}(\langle\boldsymbol{\delta} \phi \boldsymbol{\delta} \boldsymbol{\phi}\rangle \circ\langle\boldsymbol{\delta} \boldsymbol{\phi} \boldsymbol{\delta} \boldsymbol{\phi}\rangle) \\
& \approx-\frac{3}{4} \lambda\left[\prec \delta \phi_{\|}^{2} \succ^{2}+2 \prec \delta \phi_{\|}^{2} \succ \prec \delta \phi_{\perp}^{2} \succ+5 \prec \delta \phi_{\perp}^{2} \succ^{2}\right] \equiv \delta V_{T}
\end{aligned}
$$

Here $\langle\cdot\rangle_{G}$ denotes the gaussian approximation to the evaluation of the average. The last line arises if the spatial averages are approximated by the corresponding thermal averages, as is expected to be accurate when $L$ exceeds the correlation length. The resulting quantity, $\delta V_{T}$, then depends on the particular state only through the magnitude of its order parameter, $\phi_{0}$.

With the above preparations, we are now in a position to simplify the expression (10) for the partition function. We first note that the functional integral factorizes into a regular (8-dimensional) integral over the $\mathrm{O}(4)$ order parameter $\phi$ and its time derivative $\boldsymbol{\psi}$ and a functional integral over the field fluctuation $\delta \phi(\overline{\boldsymbol{r}})$ and its time derivative $\bar{\delta} \boldsymbol{\psi}(\boldsymbol{r})$,

$$
\mathcal{Z}_{T}=\int d^{4} \underline{\boldsymbol{\psi}} \mathrm{e}^{-\frac{\Omega}{T} K_{0}} \int d^{4} \underline{\boldsymbol{\phi}} \mathrm{e}^{-\frac{\Omega}{T}\left(V_{0}+\delta V_{T}\right)} \int \mathcal{D}[\boldsymbol{\delta} \boldsymbol{\phi}(\boldsymbol{r}), \boldsymbol{\delta} \boldsymbol{\psi}(\boldsymbol{r})] \mathrm{e}^{-\frac{\Omega}{T} E_{\mathrm{qp}}}
$$


The integration over $\boldsymbol{\psi}$ yields the factor $(2 \pi T / \Omega)^{2}$ which depends only on temperature. The integral over the quasiparticle degrees of freedom yields the conditional quasiparticle partition function $\mathcal{Z}_{\mathrm{qp}}$. Using the Bose-Einstein values for the mode occupancies $n_{k}^{(j)}$, we find $\ln \mathcal{Z}_{\mathrm{qp}}=\ln \mathcal{Z}_{\mathrm{qp}}^{\|}+3 \ln \mathcal{Z}_{\mathrm{qp}}^{\perp}$, where

$$
\begin{aligned}
\ln \mathcal{Z}_{\mathrm{qp}}^{(j)} & =\sum_{\mathbf{k} \neq \mathbf{0}} \ln \bar{n}_{k}^{(j)}=-\sum_{\mathbf{k} \neq \mathbf{0}} \ln \left[1-\mathrm{e}^{-\omega_{k}^{(j)} / T}\right] \\
& =-\frac{1}{T} \sum_{\mathbf{k} \neq \mathbf{0}} \omega_{k}^{(j)} n_{k}^{(j)}+\sum_{\mathbf{k} \neq \mathbf{0}}\left[\bar{n}_{k}^{(j)} \ln \bar{n}_{k}^{(j)}-n_{k}^{(j)} \ln n_{k}^{(j)}\right] \\
& =-\frac{\Omega}{T}\left(V_{\mathrm{qp}}^{(j)}-T S_{\mathrm{qp}}^{(j)}\right)
\end{aligned}
$$

for each chiral component $j$ (with $\bar{n}_{k}^{(j)} \equiv 1+n_{k}^{(j)}$ ). The total energy density of the quasiparticles is then $V_{\mathrm{qp}}=V_{\mathrm{qp}}^{\|}+3 V_{\mathrm{qp}}^{\perp}$ and their entropy density is $S_{T}=S_{\mathrm{qp}}^{\|}+3 S_{\mathrm{qp}}^{\perp}$. Consequently, we may write the overall partition function on a simple form,

$$
\mathcal{Z}_{T}=\left(\frac{2 \pi T}{\Omega}\right)^{2} \int d^{4} \underline{\phi} \mathrm{e}^{-\frac{\Omega}{T} F_{T}}=\left(\frac{2 \pi T}{\Omega}\right)^{2} 4 \pi \int_{0}^{\infty} \phi_{0}^{3} d \phi_{0} \int_{0}^{\pi} \sin ^{2} \chi_{0} d \chi_{0} \mathrm{e}^{-\frac{\Omega}{T} F_{T}}
$$

where $F_{T}=V_{T}\left(\phi_{0}, \chi_{0}\right)-T S_{T}\left(\phi_{0}\right)$ is the effective free energy density, with the effective potential energy density being $V_{T}=V_{0}\left(\phi_{0}, \chi_{0}\right)+V_{\mathrm{qp}}\left(\phi_{0}\right)+\delta V_{T}\left(\phi_{0}\right)$.

It should be noted that the $\chi_{0}$ dependence of the free energy is only through the term $-H \phi_{0} \cos \chi_{0}$ in the bare potential $V_{0}$. For $H>0$ this term has its minimum value for $\chi_{0}=0$ and it increases steadily until reaching its maximum for $\chi_{0}=\pi$. All stationary points of $F_{T}$ are therefore located on the $\sigma$ axis and occur where $\left(\partial F_{T} / \partial \sigma_{0}\right)_{T}$ vanishes.

\section{Phase Structure}

Once the expression for the partition function has been derived, it is possible to discuss the statistical properties of the chiral order parameter $\phi$. The structure of the free energy density is illustrated in Fig. 3 in the thermodynamic limit $(L \rightarrow$ $\infty$ ) for both the $\mathrm{O}(4)$ symmetric case (vanishing $m_{\pi}$ ) and the realistic case. The equilibrium points can be identified as those where the free energy density $F_{T}$ is stationary. Using the expression for the free energy derived above, together with the self-consistent dispersion relations, it is elementary to show that

$$
\left(\frac{\partial F_{T}}{\partial \sigma_{0}}\right)_{T}=\mu_{0}^{2} \sigma_{0}-H
$$

Comparing this relation with Eq. (2), we see that statistical equilibrium (stationary free energy) and dynamical equilibrium (no acceleration of the order parameter) indeed occur simultaneously, as required by thermodynamic consistency. Therefore, the phase diagram can be determined already on the basis of Eq. (2). 

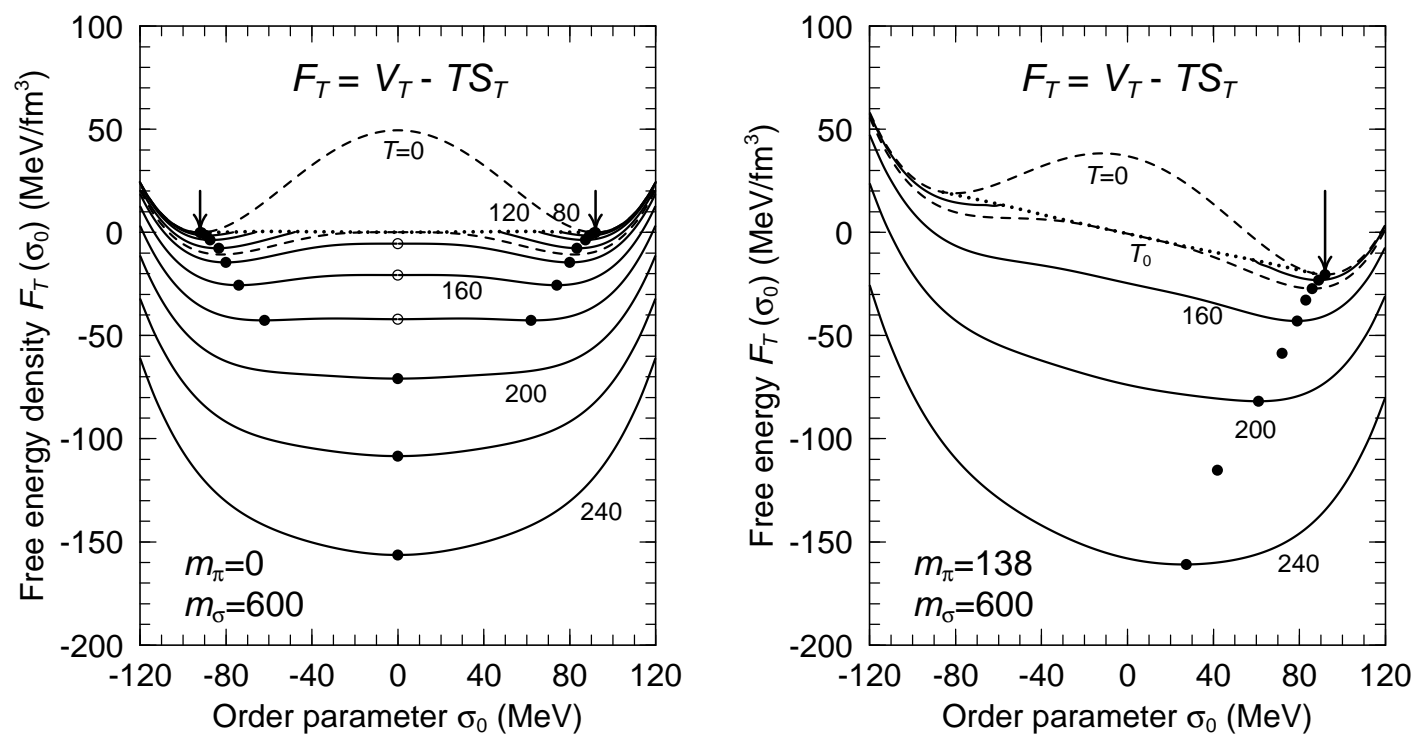

FIGURE 3. Left panel (3a): The free energy density $F_{T}$ as a function of the order parameter $\sigma_{0}$ for various values temperatures $T$, for the idealized $\mathrm{O}(4)$ symmetric case $\left(m_{\pi}=0\right)$. At each $T$, the solid (or open) dots indicate the favored (or unfavored) equilibria. The result for $T=T_{0}=\sqrt{2} v$ is indicated by a dashed curve. For $T<T_{0}$, the order parameter must exceed a certain minimum value before all quasiparticle modes are stable and the corresponding end points are connected by the dotted curve. The top dashed curve shows the bare potential $V_{0}$ that arises when $T=0$. Right panel (3b): The corresponding result for the realistic case $\left(m_{\pi}=138 \mathrm{MeV}\right)$.

The resulting equilibria are traced out in Fig. 4a for four different values of the free pion mass. In the idealized case of vanishing pion mass, the ground-state minimum in $F_{T}$ is located at $\sigma_{0}=f_{\pi}$, where $F_{T}$ vanishes, $F_{T}^{\mathrm{vac}}=0$. The minimum in $F_{T}$ moves inwards as $T$ is increased, at first very slowly and then progressively faster, until a certain temperature is reached, $T=T_{1}$. At this temperature, the stationary points in $F_{T}$ turn from minima to maxima as the tracing curve continues downwards towards the critical point.

Once the temperature exceeds the critical value $T_{0}$, a solution to the gap equations exist for all values of $\phi_{0}$, and the free energy has a minimum at $\phi_{0}=0$. Thus, in the temperature range between $T_{0}$ and $T_{1}$, there are two minima in $F_{T}$, separated by a maximum that is only slightly higher than the symmetric minimum. The outer minimum generally carries a larger statistical weight (the value of $F_{T}$ is lower). Consequently, it represents the thermodynamcially preferred state and the symmetric configuration is merely metastable (with the maximum representing an unstable equilibrium). We therefore refer to $T_{1}$ as the transition temperature. Above $T_{1}$ there the only stationary point is the minimum at symmety, $\phi_{0}=0$.

This general structure of the free energy, as obtained with the semi-classical treatment, implies that the system will display a first-order phase transition at $T=T_{1}$, with the preferred magnitude of the order parameter dropping abruptly 

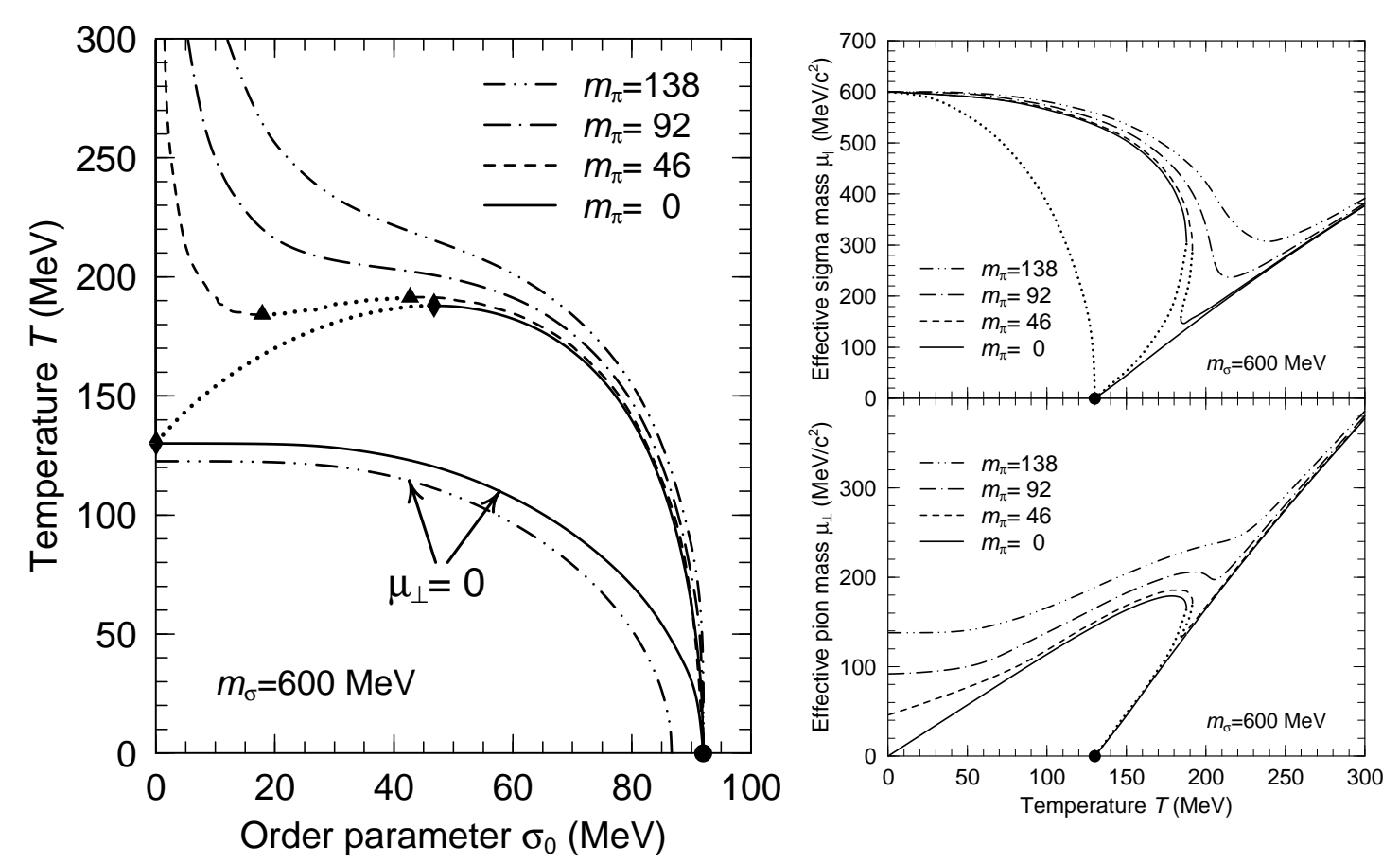

FIGURE 4. Left panel (4a): The stable and unstable (dotted) equilibrium values of the chiral order parameter $\sigma_{0}=\phi_{0} \cos \chi_{0}$ at a given quasiparticle temperature $T$, as traced on the chiral phase diagram for the indicated four values of the pion mass $m_{\pi}$. For $m_{\pi}=0$ the high-temperature $\mathrm{O}(4)$ symmetric equilibrium branch extends upwards along the vertical axis from the critical point marked by the diamond. The critical boundary defined by $\mu_{\perp}=0$ (within which the field is supercritical) is delineated for $m_{\pi}=0$ and $138 \mathrm{MeV}$. The solid dot is located at the common ground state, $\sigma_{0}=f_{\pi}=92 \mathrm{MeV}$. Right panel (4b): The equilibrium values of the effective masses $\mu_{\|}$(top) and $\mu_{\perp}$ (bottom) for $\sigma$-like and $\pi$-like quasiparticles, respectively, as functions of the temperature $T$, for the same four different pion masses $m_{\pi}$; the values at the unstable equilibria are traced by the dotted curves. In addition, the top panel shows the value of $\mu_{\|}$along the critical boundary for $m_{\pi}=0$ (dotted curve on far left).

from a fairly large finite value to zero. However, as is evident from Fig. 3a, the metastable minimum at $\phi_{0}=0$ is very shallow and a finite system would therefore display large fluctuations in $\phi_{0}$ (see Fig. 5). Moreover, the use of a finite pion mass will erode this phase structure, as the free-energy curves will be tilted towards the positive $\sigma$ direction (Fig. 3b). The outer minimum will then occur only on $\sigma$ axis (at $\sigma_{0}=f_{\pi}$ ), while there will be a saddle point situated in the opposite direction. As seen in Fig. 4a, when $m_{\pi}$ exceeds about half the physical value, the resulting tilting suffices to eliminate the symmetric minimum altogether and there is then only a single minimum at any temperature (located on the positive part of the $\sigma$ axis). From that value on, the system displays a smooth crossover from a strongly broken to a weakly broken phase, with the most rapid change in order parameter occurring at temperatures somewhat above $200 \mathrm{MeV}$. 
The phase structure discussed above is reflected in the behavior of the equilibrium values of the effective quasiparticle masses, as shown in Fig. 4b. When the specified value of $m_{\pi}$ is sufficiently small, the associated phase transition causes a backbend in the mass curves, as the system is traced through the unstable equilibrium branch. For perfect $\mathrm{O}(4)$ symmetry $\left(m_{\pi}=0\right)$, the backbend reaches all the way to the critical point where all the effective masses vanish, $\mu_{0}=\mu_{\perp}=\mu_{\|}=0$, and from there on they are exactly degenerate and grow approximately linearly with $T$. As noted above, the branch with the large masses is generally the thermodynamically preferred one. For the case of $m_{\pi}=0$, Fig. $4 \mathrm{~b}$ also shows the evolution of $\mu_{\|}$along the critical path where $\mu_{\perp}$ vanishes. It drops approximately quadratically from its free value at $T=0$ to zero at $T_{0}$.

For the larger values of $m_{\pi}$, the evolution is smooth, but the basic two-phase structure of the system remains visible as a distinct minimum in the $\sigma$ mass. (The location of this minimum may be used as an indicator for the effective transition temperature [15].) At higher temperatures, the masses become nearly degenerate as chiral symmetry is approached.

\section{Finite Size}

Since the systems available for actual experimental study have a rather limited spatial extension, it is important to assess the importance of finite-size effects. As it turns out [15], the free energy density $F_{T}$ depends very little on the actual volume $\Omega$, once the side length $L$ is above $5 \mathrm{fm}$ or so, and it makes no practical difference whether it is calculated in terms of the quantized modes, as indicated above, or in the continuum limit. Thus the size dependence of the statistical properties arises primarily through the volume $\Omega$ multiplying $F_{T}$ in the integrand in (20).

When the system is small, the integrand in the partition function is no longer narrowly peaked at the minima in $F_{T}$. Rather, it represents a probability density in the $\mathrm{O}(4)$ space of the order parameter $\phi$. Since $F_{T}$ depends only on the magnitude of the order parameter, $\phi_{0}$, and its angle of disorientation, $\chi_{0}$, it is instructive to project onto the plane of those two state variables,

$$
\mathcal{Z}_{T} \sim \int_{0}^{\infty} 4 \pi \phi_{0}^{3} d \phi_{0} \int_{0}^{\pi} \sin ^{2} \chi_{0} d \chi_{0} \mathrm{e}^{-\frac{\Omega}{T} F_{T}\left(\phi_{0}, \chi_{0}\right)}=\int_{0}^{\infty} d \phi_{0} \int_{0}^{\pi} d \chi_{0} W_{T}\left(\phi_{0}, \chi_{0}\right)
$$

where $W_{T}$ is the statistical weight for finding the system with an order parameter having the specified magnitude and disorientation.

Generally speaking, the statistical fluctuations in a finite system tends to wash out the sharp phase structure characteristic of infinite matter. The importance of this effect on the temperature dependence of the order parameter is brought out in Fig. 5. The changes are most significant in the idealized $\mathrm{O}(4)$ symmetric scenario. In particular, it can be seen that the first-order transition obtained for $m_{\pi}=0$ becomes less prominent as the volume is decreased to realistic sizes and it has disappeared altogether for the smallest volume considered ( $L \approx 6 \mathrm{fm}$ ). 

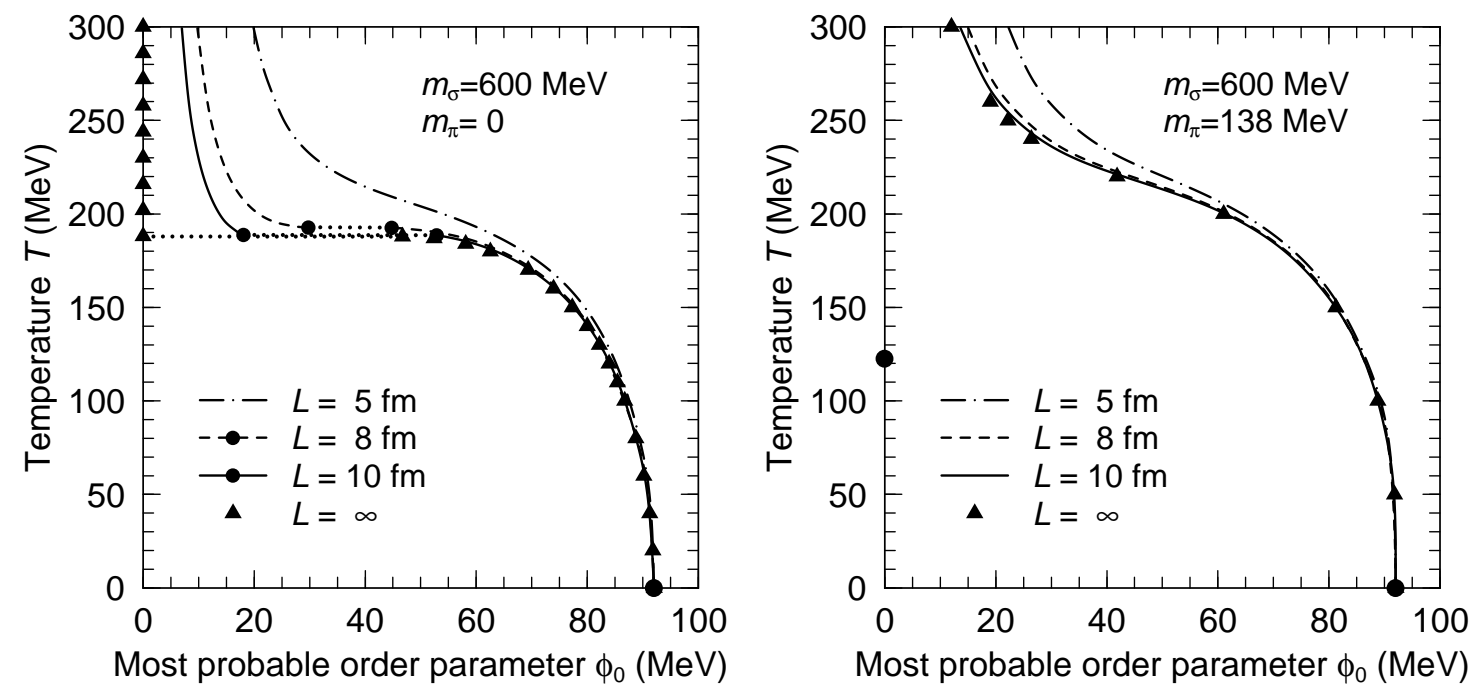

FIGURE 5. The most probable value of the magnitude of order parameter, $\phi_{0}$, as a function of the temperature $T$, for various values of the side length $L$ of the cubic volume considered, with either $m_{\pi}=0$ (left panel: $5 \mathrm{a}$ ) or $m_{\pi}=138 \mathrm{MeV}$ (right panel: $5 \mathrm{~b}$ ).

Such qualitative change does not occur when a realistic value of $m_{\pi}$ is employed, since the behavior is then already smooth for large systems. In fact, the finite-size effect is hardly visible until rather small volumes are reached. Furthermore, for such small volumes the difference between the results obtained for the various specified values of $m_{\pi}$ is less noticeable. This finding suggests that a quantitative extraction of the matter equation of state from analysis of the small finite systems involved in actual experiments depends heavily on the availability of reliable models.

Another important finite-size effect is the fluctuation in the $\mathrm{O}(4)$ orientation of the order parameter $\phi$. However, because of the large entropy carried by the quasiparticles, even a relatively modest change in the disalignment angle $\chi_{0}$ leads to a strong reduction in the statistical weight. As a result, the equilibrium distribution $P(\underline{\phi})$ remains fairly confined around the positive $\sigma$ direction, as is illustrated in Fig. 6. Thus, the idealized "sombrero" picture in which the order parameter has a fairly isotropic distribution at high temperatures may be somewhat misleading.

\section{Sampling of Thermal Field Configurations}

For actual numerical computations, it is often of interest to sample the field from a suitably characterized ensemble and we outline how it is possible to devise a fast, efficient, and robust method for sampling from the thermal ensemble [15].

The first task is to sample the order parameter in accordance with the statistical weight $W_{T}(\underline{\phi}, \underline{\boldsymbol{\psi}})$. Since the time derivative $\underline{\psi}$ is governed by a four-dimensional normal distribution which is isotropic and entirely decoupled from the other degrees of freedom (see Eq. (16)), it is elementary to sample this quantity. 


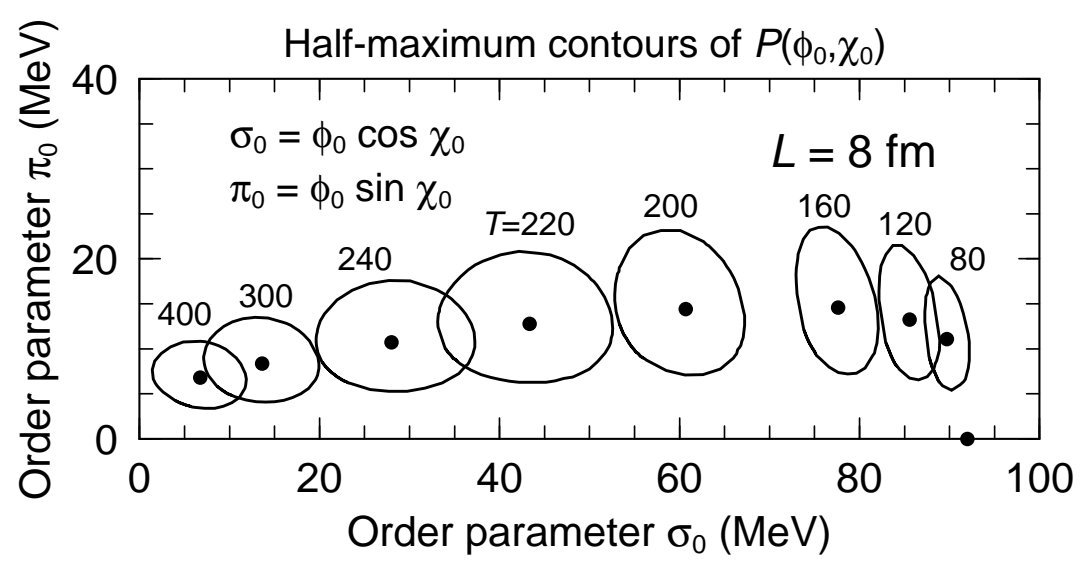

FIGURE 6. The projected equilibrium distribution of the chiral order parameter, $P\left(\phi_{0}, \chi_{0}\right) \sim \phi_{0}^{3} \sin ^{2} \chi_{0} W_{T}(\underline{\phi})$, displayed as a function of its aligned component, $\sigma_{0}=\phi_{0} \cos \chi_{0}$, and the magnitude of its transverse component, $\pi_{0}=\phi_{0} \sin \chi_{0}$, for a cubic box of side length $L=8 \mathrm{fm}$. For each temperature $T$, the solid dot indicates the location of the maximum in $P\left(\sigma_{0}, \pi_{0}\right)$ and the solid curve traces out the half-maximum contour, as obtained by scaling the continuum results down to the appropriate finite volume $\Omega=L^{3}$.

It is more complicated to sample the magnitude $\phi_{0}$, due to the intricate structure of its probability distribution, as discussed above. However, the numerical effort required is quite modest. The most efficient method utilizes a precalculation of the effective masses as functions of $\phi_{0}$, for the particular $T$ of interest. The $\chi_{0^{-}}$ independent part of the effective potential, $V_{T}\left(\phi_{0}, 0\right)$, can then be obtained together with the corresponding entropy $S_{T}\left(\phi_{0}\right)$. Since the dependence on $\chi_{0}$ is simple, an exact integration over $\chi_{0}$ is possible, and so, in effect, the probability distribution for $\phi_{0}$ can be pretabulated. It is then a numerically trivial task to sample $\phi_{0}$.

Once the magnitude $\phi_{0}$ has been selected, it is straightforward to sample the disalignment angle $\chi_{0}$ (using either its exact form or its Gaussian approximation). In order to orient $\underline{\phi}$ in the $\pi$ subspace, there remains the task of selecting the remaining $O(3)$ spherical angles $\vartheta_{0}$ and $\varphi_{0}$, upon which the order parameter is given by $\boldsymbol{\phi}=\left(\phi_{0} \cos \chi_{0}, \phi_{0} \sin \chi_{0} \sin \vartheta_{0} \cos \varphi_{0}, \phi_{0} \sin \chi_{0} \sin \vartheta_{0} \sin \varphi_{0}, \phi_{0} \sin \chi_{0} \cos \vartheta_{0}\right)$.

Since the different quasi-particle modes can be regarded as effectively decoupled, their sampling is best done by making an expansion into the elementary modes,

$$
\delta \phi_{\|}(\boldsymbol{r}, t)=\left(\frac{2}{\Omega}\right)^{\frac{1}{2}} \sum_{\mathbf{k} \neq \mathbf{0}} C_{\mathbf{k}}^{\|} \cos \left(\mathbf{k} \cdot \boldsymbol{r}-\omega_{k}^{\|} t-\eta_{\mathbf{k}}^{\|}\right),
$$

and similarly for the three transverse chiral components $\delta \phi_{\perp}(\boldsymbol{r}, t)$. The phase $\eta_{\mathbf{k}}$ is random in the interval $(0,2 \pi)$ and is thus trivial to sample. Furthermore, the real (and positive) amplitude $C_{\mathbf{k}}$ can be related to the number of quanta $n_{\mathbf{k}}$ by considering the energy carried by the mode, $E_{\mathbf{k}}=n_{\mathbf{k}} \omega_{k}=\omega_{k}^{2} C_{\mathbf{k}}^{2}$. By this token, the problem has been reduced to sampling the number of quanta $n_{\mathbf{k}}$ which is elementary. [In fact, $n_{\mathbf{k}}$ can be regarded as counting the number of successive times 
the sampling of a standard random number yields a value below $\left.\exp \left(-\epsilon_{k} / T\right)\right)$.] The thermal average of $n_{\mathbf{k}}$ is equal to the occupancy $f_{k}$ employed in the calculation of the entropy, $\prec n_{\mathbf{k}} \succ=f_{k}$. It may be noted that while the energy relation employed is the classical one (which omits the zero-point contribution), the occupation number is properly quantized and sampled in accordance with quantum statistics. This method eliminates divergencies while retaining the essential quantum fluctuations.

Once the quasiparticle amplitudes and phases have been selected, the expansion (23) readily yields the value of the field fluctuations, $\delta \boldsymbol{\phi}(\boldsymbol{r})$ at a specified time $t_{0}$. The corresponding conjugate momentum $\delta \boldsymbol{\psi} \equiv \partial_{t} \boldsymbol{\delta} \phi$ readily follows,

$$
\delta \psi_{\|}(\boldsymbol{r}, t)=\left(\frac{2}{\Omega}\right)^{\frac{1}{2}} \sum_{\mathbf{k} \neq \mathbf{0}} \omega_{k}^{\|} C_{\mathbf{k}}^{\|} \sin \left(\mathbf{k} \cdot \boldsymbol{r}-\omega_{k}^{\|} t-\eta_{\mathbf{k}}^{\|}\right)
$$

When the equations of motion are propagated by a leap-frog method, the field strength $\delta \boldsymbol{\phi}(\boldsymbol{r})$ is calculated at $t_{N}=N \Delta t$ while the momentum $\delta \boldsymbol{\psi}(\boldsymbol{r})$ is obtained at the mid points. The appropriate initial $\delta \boldsymbol{\psi}(\boldsymbol{r})$ can then easily be obtained by evaluating (24) at $t=t_{0}+\frac{1}{2} \Delta t$ after $C_{\mathbf{k}}$ and $\eta_{\mathbf{k}}$ have been selected at $t_{0}$.

Finally, the system in which the sampling has been done is aligned with the order parameter $\phi$ whose $\mathrm{O}(4)$ direction is $\left(\chi_{0}, \vartheta_{0}, \varphi_{0}\right)$ (the mass tensor is diagonal in this system). Therefore a corresponding rotation is required to express the sampled field configuration with respect to the chiral reference frame $\left(\hat{\sigma}, \hat{\pi}_{1}, \hat{\pi}_{2}, \hat{\pi}_{3}\right)$.

\section{Correlation Function}

It is interesting to calculate the correlation function of the chiral field since this quantity determines the spectral distribution of the emitted field quanta. The density matrix for the quasi-particle field is a $4 \times 4$ tensor,

$$
\boldsymbol{C}\left(r_{12}, t_{12}\right) \equiv \prec \boldsymbol{\delta} \phi\left(\boldsymbol{r}_{1}, t_{1}\right) \boldsymbol{\delta} \boldsymbol{\phi}\left(\boldsymbol{r}_{2}, t_{2}\right) \succ
$$

where the average is over the thermal ensemble held at the temperature $T$. Since an ensemble in equilibrium is invariant in time, the correlation function depends only on the time difference $t_{12}=t_{1}-t_{2}$. Moreover, translational symmetry implies that the spatial dependence is via the separation $\boldsymbol{r}_{12}=\boldsymbol{r}_{1}-\boldsymbol{r}_{2}$ and invariance under spatial rotations ensures that only the magnitude $r_{12}=\left|\boldsymbol{r}_{12}\right|$ enters.

Utilizing the expansion (23), it is elementary to show that the correlation tensor $\boldsymbol{C}$ is diagonal with the elements $C_{\|}$and $C_{\perp}$, where

$$
C_{\|}(r, t)=\frac{1}{\Omega} \sum_{\mathbf{k} \neq \mathbf{0}} \frac{1}{\omega_{k}^{\|}} \frac{\cos \left(\mathbf{k} \cdot \boldsymbol{r}-\omega_{k}^{\|} t\right)}{\mathrm{e}^{\omega_{k}^{\|} / T}-1} \asymp \frac{1}{2 \pi^{2} r} \int_{\mu_{\|}}^{\infty} d \omega \frac{\sin k r}{\mathrm{e}^{\omega / T}-1} \cos \omega_{k}^{\|} t,
$$

and similarly for $C_{\perp}(\boldsymbol{r}, t)$. Since $f_{k} / \omega_{k}$ is equal to the thermal average $\prec C_{\mathbf{k}}^{2} \succ$, we recognize the familiar result (see Eq. (146.10) in Ref. [18], for example). 

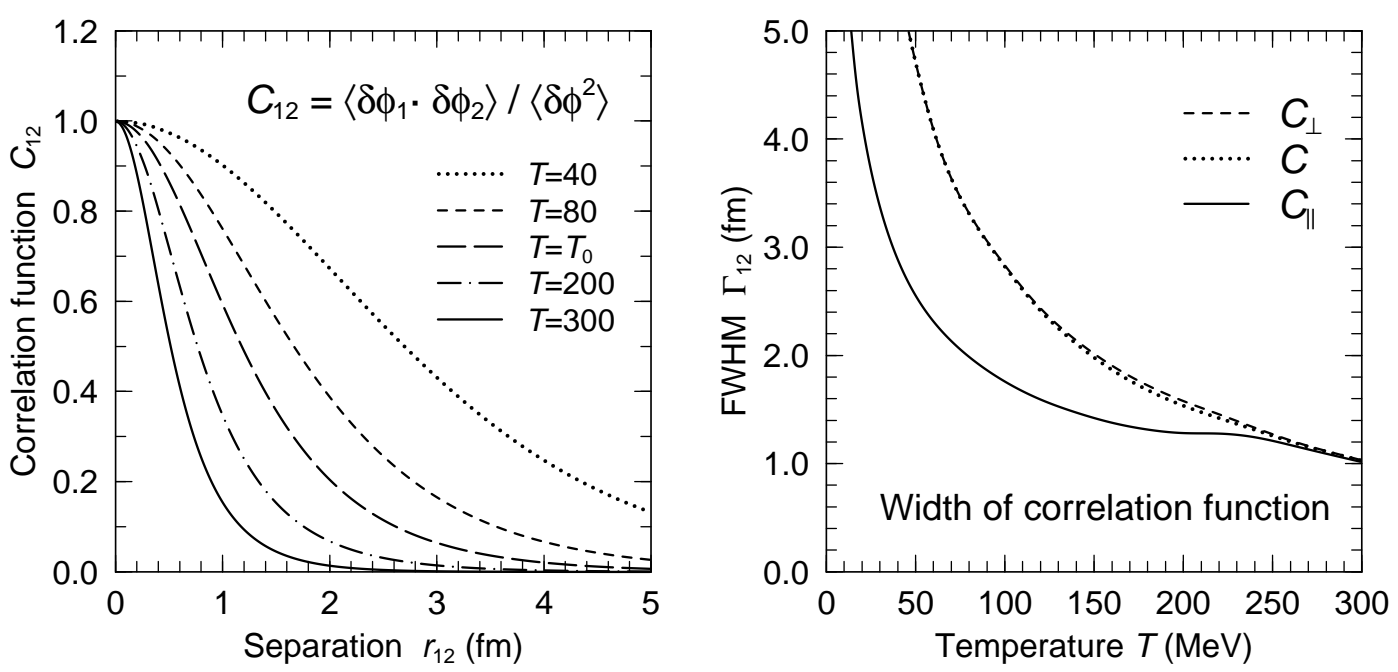

FIGURE 7. Correlation function in equilibrium. Left (7a): The reduced correlation function $C_{12}$ for a range of temperatures $T$, employing for each $T$ the most probable magnitude of the order parameter, $\phi_{0}$. Right (7b): The FWHM correlation length $\Gamma_{12}$ of $C_{12}$ as a function of $T$, for the total field fluctuations as well as the transverse and parallel components separately.

The usual correlation function is the trace of $\boldsymbol{C}$,

$$
C \equiv \prec \boldsymbol{\delta} \phi\left(\boldsymbol{r}_{1}, t_{1}\right) \cdot \boldsymbol{\delta} \phi\left(\boldsymbol{r}_{2}, t_{2}\right) \succ=\operatorname{tr} \boldsymbol{C}=C_{\|}\left(r_{12}, t_{12}\right)+3 C_{\perp}\left(r_{12}, t_{12}\right) .
$$

Its overall magnitude is set by its value at zero, which is simply the corresponding variance in the field strength, $C(0,0)=\prec \delta \phi^{2} \succ$. It is instructive to consider the reduced function $C_{12} \equiv C\left(r_{12}, 0\right) / \prec \delta \phi^{2} \succ$ which is unity for $\boldsymbol{r}_{1}=\boldsymbol{r}_{2}$. In general, $C_{12} \sim\left(1 / r_{12}\right) \exp \left(-\mu r_{12}\right)$ in the limit of large separations, $r_{12} \rightarrow \infty$, so that $1 / \mu c$ provides a simple measure of the correlation length. In the special case when the effective mass vanishes, the reduced equal-time correlation function is given on analytical form, $C_{12} \asymp(3 / \zeta)(\operatorname{coth} \zeta-1 / \zeta)$, where $\zeta \equiv \pi T r_{12}$. In this extreme case, the correlation function falls off only as $\sim 1 / r_{12}$.

Figure 7 a shows how the reduced correlation function evolves with temperature in equilibrium. At high $T$ we have $\mu \sim T$, so then the correlation length tends to zero. For temperatures below critical, the field fluctuations are predominantly associated with the transverse modes, since those have the smallest effective mass, $\mu_{\perp} \ll \mu_{\|}$, and the correlation length grows ever larger.

It is convenient to characterize $C_{12}$ by its full width at half maximum, $\Gamma_{12}$, since this quantity is always possible to extract, even when the mass vanishes. Figure $7 \mathrm{~b}$ shows this measure of the correlation length as a function of temperature, using again the most probable value of $\phi_{0}$. For temperatures near and below $T \approx 200 \mathrm{MeV}$ the dominant fluctuations are perpendicular to the order parameter since the corresponding effective mass is relatively small. For higher temperatures the asymptotic regime is approached where the chiral symmetry is approximately restored and the fluctuations are similar in all four chiral directions. 


\section{DYNAMICS AND OBSERVABLES}

The equilibrium properties of the system discussed above provide a useful reference for understanding the key features of its dynamics, since the system generally seeks to reestablish equilibrium in response to an external disturbance. The environment produced in a high-energy collision is characterized by a rapid cooling, driven primarily by the explosive expansion, and this forces the system away from equilibrium. The overall evolution is then the combined result of those two opposite effects. it is then a delicate task to predict the outcome and one must generally resort to dynamical calculations.

Since the evolving system is in a non-equilibrium state, it is harder to analyze. Fortunately, it is possible to depict a general field configuration on a equal footing with the equilibrium configurations by employing a modified phase diagram in which the degree of agitation is represented by the the average field fluctuation, $\Delta \phi=\langle\boldsymbol{\delta} \phi \circ \delta \boldsymbol{\phi}\rangle^{1 / 2}$, rather than the quasiparticle temperature $T$ (as in Figs. 4a and $5)$. Since $\Delta \phi$ is meaningful for any individual field configuration, it is thus possible to project an arbitrary state onto the generalized diagram (which is occasionally referred to as the "chiral road map"). The standard phase diagram (the curves in Fig. 4a obtained for $m_{\pi}=138 \mathrm{MeV}$ ) then appears as shown in Fig. 8a below.
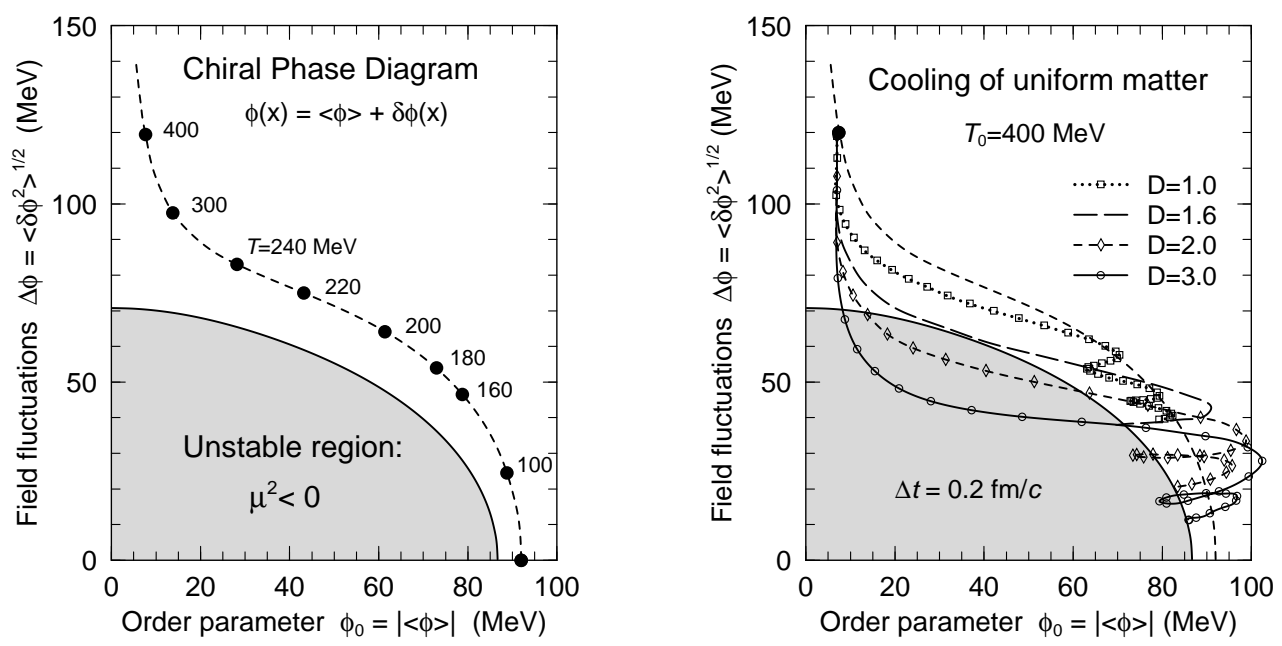

FIGURE 8. Left panel (8a): The equilibrium path on the "chiral road map" in which the field configuration is projected onto a single point, $\phi(r) \rightarrow\left(\phi_{0}, \Delta \phi\right)$. The adiabatic path of equilibrium points (dashed curve), which represents the major "highway", exhibits a gradual cross over from the broken phase at low temperature to the nearly restored phase at high temperature. The shaded area covers the classically forbidden region within which the quasipions are subject to a supercirtical effective field. Right panel (8b): The dynamical paths of systems that have been prepared in equilibrium at $T_{i}=400 \mathrm{MeV}$ and then subjected to a Rayleigh cooling with strengths $D$ adjusted to emulate scaling expansions ( $D=1$ emulates a longitudinal Bjorken expansion). [9] 


\section{Emulation of Scaling Expansions}

It is expected that the early parton dynamics causes the chiral field to be in a state of rapid expansion. The subsequent evolution may then lead to a supercooled configuration situated inside the unstable region, thus effectively producing a "quench". A number of quenched scenarios have been considered [6,19-21,7,22,23] but they were imposed by fiat, thereby reducing the predictive power of the dynamcial calculations (essentially any degree of magnification can be achieved by suitable adjustment of the initial conditions). The degree of arbitrariness can be reduced by elucidating under which conditions a quench-like early scenario may develop dynamically from plausible initial configurations.

Bjorken-like scaling expansions [24] provide especially simple expansion scenarios. Both purely longitudinal $[19,7,22,23,13]$ and fully isotropic scaling expansions $[20,25]$ have been treated. In order to achieve a global impression of the influence of expansion, it suffices to augment the equation of motion (1) by a term emulating a scaling expansion in $D$ dimensions, $-(D / t) \partial_{t} \phi$. This term has a form akin to the Rayleigh dissipation function in classical mechanics and it acts as a time-dependent damping that reduces the field fluctuations in the course of time. In order to examine its effect, one may ignore the spatial geometry and consider a macroscopically uniform configuration within a large box. The discussion is then simplified and the resulting scenarios can be regarded as idealized representations of chiral matter subjected to an externally prescribed cooling rate, and so the results will have a corresponding general applicability.

Figure $8 \mathrm{~b}$ shows dynamical trajectories obtained in this manner for $D=1-3$. The effect increases with $D$, since the dimensionality of the expansion effectively acts as the strength of the damping term. The isotropic expansion $(D=3)$ leads to a significant incursion into the unstable region, while the longitudinal expansion $(D=1)$ keeps the system well within the stable region.

Utilizing Eqs. (5-6) it is possible to extract the time dependence of the effective masses and their evolution will reflect the behavior exhibit by the dynamical paths depicted in Fig. 8b. In particular, the incursions into the supercritical region leads to negative values of $\mu_{\perp}^{2}$ and a corresponding exponential growth of those modes for which $\mu_{\perp}^{2}+k^{2}<0$. In fact, the most important effects of the dynamics can be well understood on the basis of the behavior of the quaisparticle mass tensor [9], as will be illustrated later.

It is instructive to see how the various cooling scenarios affect the pion observables. For this purpose, a Fourier decomposition of the pion field is useful,

$$
\boldsymbol{\pi}(\boldsymbol{r}, t)=\sum_{\mathbf{k}} \boldsymbol{\pi}_{\mathbf{k}}(t) \mathrm{e}^{i \mathbf{k} \cdot \mathbf{r}}
$$

Due to the presence of the cooling term, the amplitudes tend towards zero as

$\boldsymbol{\pi}_{\mathbf{k}}(t) \sim t^{-D / 2}$ at late times. Consequently, the field decouples into free modes and it is then meaningful to extract the asymptotic values of the observables. 
Of particular interest is the spectral distribution of the pions and Fig. 9a shows their asymptotic power spectrum as a function of their kinetic energy $\omega_{k}-m_{\pi}$,

$$
P_{\pi}\left(E_{\mathrm{kin}}\right) \sim \sum_{\mathbf{k} \neq \mathbf{0}} \omega_{k}\left|\sqrt{\frac{\omega_{k}}{2}} \pi_{\mathbf{k}}+\frac{i}{\sqrt{2 \omega_{k}}} \dot{\pi}_{\mathbf{k}}\right|^{2} \delta\left(E_{\mathrm{kin}}+m_{\pi}-\omega_{k}\right) .
$$

It is clear that the incursions into the supercritical region for $D=2,3$ lead to dramatic enhancements of the yield in the lowest energy bin, $E_{\text {kin }}<200 \mathrm{MeV}$, whereas there is hardly any visible effect for $D=1$.

The dynamically induced enhancement of the soft pion modes is also reflected in their resulting correlation function $C_{\pi}\left(r_{12}\right)$, as shown in Fig. 9b. While the correlation function approximately retains its initial thermal form for $D=1$, it widens steadily for the larger cooling rates, as the increased strength of the softest pion modes causes it to acquire a pronounced tail.

Such analyses show that the occurrence of instabilities and the associated amplification of pionic modes depend sensitively on the cooling rate, which in turn is intimately related to the character of the expansion. The idealized scenario for $D=3$ corresponds closely to the isotropic expansion considered in Refs. [20,25] and the results corroborate the conclusion in Ref. [20] that such a scenario leads to amplification. Furthermore, the analysis suggest that a longitudinal expansion alone is insufficient to cause a quench, if the initial fluctuations are of thermal magnitude. This is consistent with what was found in Refs. [19,23] for effectively one-dimensional expansions. This qualitative sensitivity to the collision dynamics underscores the importance of employing physically reasonable initial conditions for the dynamical simulations of DCC formation.
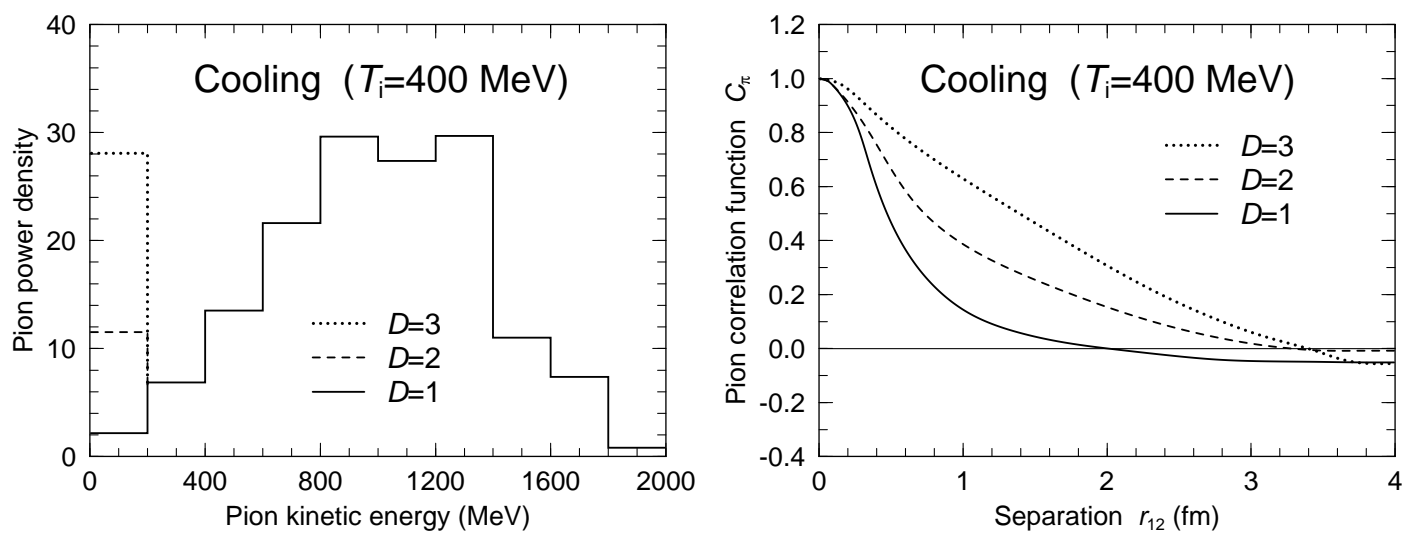

FIGURE 9. Left panel (9a): The power spectrum of the pions emerging asymptotically as a result of propagating 100 field configurations (sampled from the thermal distribution at $T_{i}=400 \mathrm{MeV}$ ) with the equation of motion (1) augmented with a Rayleigh dissipation term of strength $D=1,2,3$. Right panel (9b): The corresponding pion correlation function normalized to $C_{\pi}=1$ at $r_{12}=0$. (These results are from Ref. [26].) 


\section{Neutral Pion Fraction}

It was noted early on that isospin-directed oscillations of the pion field will result in an anomalous behavior of the neutral pion fraction $f=n_{\pi_{0}} / n_{\pi}$ [27-31]. Indeed, the distribution would be given by $P(f)=1 /(2 \sqrt{f})$ in the idealized scenario where all the pions observed arise from a fully aligned source. In practice, the observed pions may originate from unrelated regions and the anomaly is then attenuated. This is illustrated in Fig. 10a which shows the result of combining pions from $N$ independent sources. A Poisson-like distribution peaked near $f=\frac{1}{3}$ emerges when there are many independent sources, or equivalently, when the distance between emission points is large in comparison with the correlation length.

In order to give a quantitative feeling for what this inherent feature amounts to in practice, we show in Fig. 10b the distribution $P(f)$ extracted from an ensemble of 100 events that have been cooled with $D=2$, as explained above. When all the pions are used for the calculation of $f$, the resulting distribution looks fairly normal, but when only pions with a kinetic energy below $200 \mathrm{MeV}$ are considered, then $P(f)$ broadens significantly and attains an anomalous form. However, its appearance still differs significantly from the ideal form, which is only reached if all the pions arise from a single mode, such as the one having $k=0$.

This important feature can also be brought out by viewing a given source at different scales, as illustrated in Fig. 11a where the total source is divided into ever smaller sources, each one leading to a separate value of $f$. As the source size shrinks, its pion field is increasingly well aligned and the associated $P(f)$ grows correspondingly more anomalous. It has therefore been proposed that wavelet-type analyses may provide a useful tool for the extraction of DCC domain structure from the experimental data [32-34].
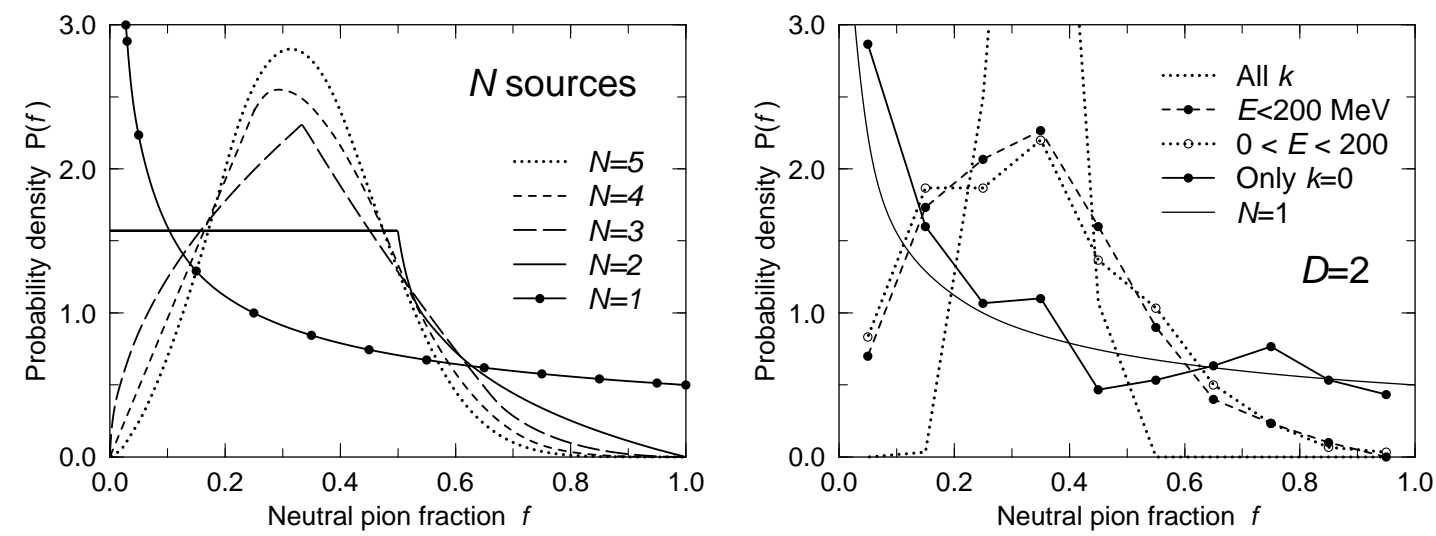

FIGURE 10. Left panel (10a): The distribution of the neutral pion fraction, $P(f)$, resulting from combining $N$ similar but independent idealized pion sources with perfect isospin alignments. Rigt panel (10b): The distribution $P(f)$ obtained when various energy cuts are applied to events obtained by subjecting field configurations prepared at $T_{i}=400 \mathrm{MeV}$ to a cooling with $D=2$. [26] 

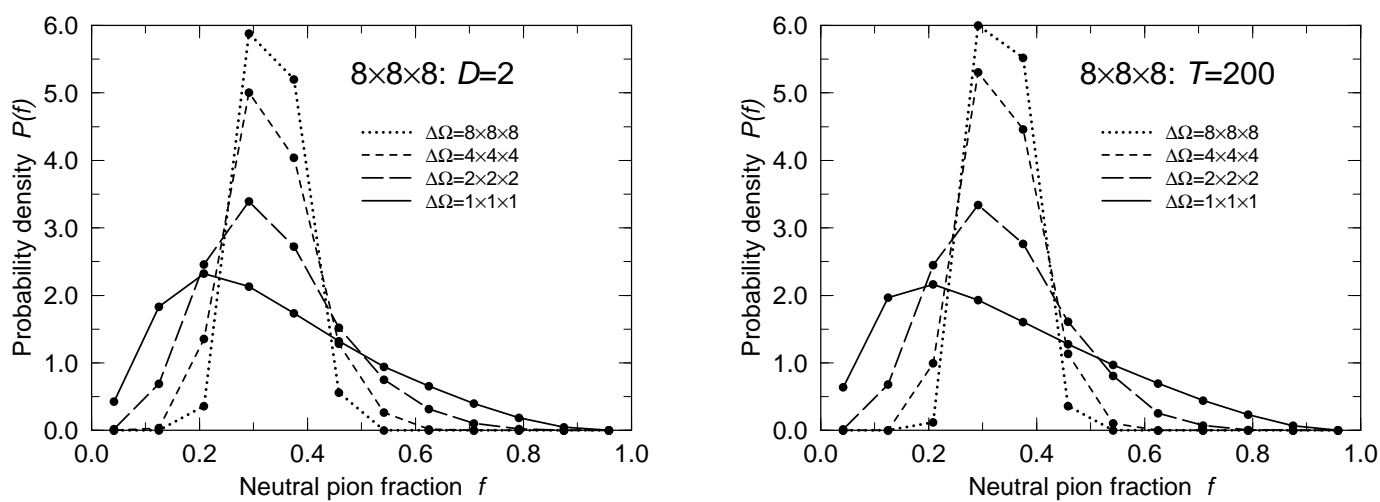

FIGURE 11. The distribution of the neutral pion fraction, $P(f)$, for a cube with side length $L=8 \mathrm{fm}$ that has been subdivided into ever smaller cubic sources. The system was either Rayleigh cooled with $D=2$ starting from $T_{i}=400 \mathrm{MeV}$ (left panel: $9 \mathrm{a}$ ) or it was prepared in equilibrium at $T=200 \mathrm{MeV}$ (right panel: $9 \mathrm{~b}$ ). (From Ref. [34].)

The key quantity determining the form of the extracted $P(f)$ is the pion correlation length. In the examples above, the growth of the correlation length was caused by the non-equilibrium evolution following a quench. However, a large correlation length can also exist in thermal equilibrium, since it grows steadily as $T$ is reduced, and thus similar results can be produced by a thermal source, as is illustrated in Fig. 11b. Therefore, the appearance of an anomalous neutral pion fraction distribution is not a unique signal of the DCC phenomenon.

\section{Dilepton Production}

Electromagnetic observables, namely dileptons [35,36] and photons [37], may provide additional information on the chiral dynamics. It is possible to extend the semi-classical treatment of the linear $\sigma$ model to the calculation of electromagnetic production processes as well, as illustrated below for the production of dileptons.

The numerical solution of the equations of motion of the chiral fields yields the evolution of the Cartesian components of the pion field, $\boldsymbol{\pi}(\boldsymbol{r}, t)$, in addition to the sigma field $\sigma(\boldsymbol{r}, t)$. The electromagnetic current density coincides with the third component of the isovector current density, $J_{\mu}(x)=\pi_{1}(x) \partial_{\mu} \pi_{2}(x)-\pi_{2}(x) \partial_{\mu} \pi_{1}(x)$, where the complex fields representing the charged pions are related to the Cartesian components by $\pi_{ \pm}(x)=\left[\pi_{1}(x) \pm i \pi_{2}(x)\right] / \sqrt{2}$.

The invariant differential dilepton yield may be calculated (to leading order in the fine structure constant $\alpha=e^{2} / 4 \pi$ ), by use of the following expression [36],

$$
\frac{d^{4} N}{d^{4} q}=\frac{2}{3 \pi}\left(\frac{\alpha}{2 \pi}\right)^{2}\left(\frac{q^{\mu} q^{\nu}}{q^{4}}-\frac{g^{\mu \nu}}{q^{2}}\right) \int d^{4} x \int d^{4} y J_{\mu}(x) \mathrm{e}^{-i q(x-y)} J_{\nu}(y)
$$




$$
=\frac{2}{3 \pi}\left(\frac{\alpha}{2 \pi}\right)^{2} \tilde{J}_{\mu}^{*}(q)\left(\frac{q^{\mu} q^{\nu}}{q^{4}}-\frac{g^{\mu \nu}}{q^{2}}\right) \tilde{J}_{\nu}(q) \text {. }
$$

This expression ignores the final-state Bose enhancement factors $1+n_{\mathbf{k}}$ which is justified when the occupation number $n_{\mathbf{k}}$ is small, as is typically the case in equilibrium. It may be noted that if the calculated pion field $\boldsymbol{\pi}(\boldsymbol{r}, t)$ is assumed to represent a standard coherent state, then the quantal evaluation of the dilepton radiation rate would lead to the above expression (30) when the commutator terms are ignored; if those commutator terms were retained, then the final-state Bose enhancement factors would be recovered.

In general, we consider an entire sample of $\mathcal{N}$ individual evolutions, $\left\{\boldsymbol{\phi}^{(n)}(\boldsymbol{r}, t)\right\}$, where the label $n$ enumerates the individual "events" in the sample. The resulting ensemble-average dilepton yield is then

$$
\prec \frac{d^{4} N}{d^{4} q} \succ=\frac{1}{\mathcal{N}} \sum_{n=1}^{\mathcal{N}} \frac{d^{4} N^{(n)}}{d^{4} q}
$$

where $d^{4} N^{(n)} / d^{4} q$ is the contribution from the particular event $n$, obtained as described above. Since we consider ensembles that have translational symmetry, the current-current correlation function, $\prec J_{\mu}(x) J_{\nu}(y) \succ$, will depend only on the spatial separation. Moreover, in the special case of an equilibrium ensemble, its temporal dependence in equilibrium is only via the time difference.

In order to verify that the adopted method indeed leads to physically reasonable results, consider the production of back-to-back dileptons from a thermal gas of free pions. In that special case the four-momentum of the dilepton is of the form $q=(M, 0)$ and we are interested in masses $M$ above $2 m_{\pi}$. The current-current contraction in (30) is then especially simple and its ensemble average is given by

$$
\prec J_{\mu}(x)\left(\frac{q^{\mu} q^{\nu}}{q^{2}}-g^{\mu \nu}\right) J_{\nu}(y) \succ=\prec \boldsymbol{J}(x) \cdot \boldsymbol{J}(y) \succ=2|\nabla C|^{2}-2 C \Delta C,
$$

where we have employed the thermal correlation function of the charged pion fields,

$$
\prec \pi_{1}(x) \pi_{1}(y) \succ=\prec \pi_{2}(x) \pi_{2}(y) \succ=C(\boldsymbol{r}, t)=\frac{1}{\Omega} \sum_{\mathbf{k}} \frac{\tilde{n}_{k}}{\omega_{k}} \cos \left(\mathbf{k} \cdot \mathbf{r}-\omega_{k} t\right),
$$

with $\tilde{n}_{k}$ being the thermal occupancy, $\tilde{n}_{k}=1 /\left(\exp \left(\omega_{k} / T\right)-1\right)$, and $(\boldsymbol{r}, t)$ denoting the difference $x-y$. [The adopted sampling procedure ensures that the numerically extracted correlation function indeed yields this expression [15].] Since the backto-back dileptons have vanishing momentum, $\mathbf{q}=\mathbf{0}$, the Fourier transform over the separation $\boldsymbol{r}$ reduces to a spatial average and we readily find

$$
\int_{\Omega} d \boldsymbol{r} \prec \boldsymbol{J}(x) \cdot \boldsymbol{J}(y) \succ=4 \int_{\Omega} d \boldsymbol{r}|\nabla C(\boldsymbol{r}, t)|^{2}=\frac{2}{\Omega} \sum_{\mathbf{k}} \frac{\tilde{n}_{k}^{2}}{\omega_{k}^{2}} k^{2}\left[1+\cos 2 \omega_{k} t\right] .
$$


The remaining Fourier transformation over the temporal difference then restricts the contributions in the sum to those modes that have frequencies $\omega_{k}$ near half the dilepton mass, $M / 2$. Thus, in the continuum limit, when both the box and the time interval are large, we recover exactly the usual expression for production of dileptons by pion annihilation,

$$
\frac{d^{4} N^{t h}}{d^{4} q d^{4} x}=\frac{4}{3 \pi}\left(\frac{\alpha}{2 \pi}\right)^{2} \int \frac{d \mathbf{k}}{(2 \pi)^{3}} \int d t \frac{\tilde{n}_{k}^{2}}{\omega_{k}^{2}} \frac{k^{2}}{M^{2}}\left[1+\cos 2 \omega_{k} t\right] \mathrm{e}^{i M t}=\frac{\alpha^{2}}{3} \frac{n_{0}^{2}}{(2 \pi)^{4}}\left(1-\frac{4 m_{\pi}^{2}}{M^{2}}\right)^{\frac{3}{2}}
$$

where $n_{0}$ denotes the occupancy of pion states having the matching frequency $\omega_{0}=M / 2$. Thus, at the formal level, the semi-classical method is indeed physically reasonable.

Figure 12a shows the dilepton production rate in a source in thermal equilibrium at $T=140 \mathrm{MeV}$, together with the corresponding result obtained when the system is prepared in a quenched configuration where nearly all the thermal energy (about $40 \mathrm{MeV} / \mathrm{fm}^{3}$ ) has been converted into potential energy of the displaced order parameter [36]. As is shown in Fig. 12b, the release of the system causes the order parameter to execute large oscillations around its equilibrium value leading to a significant enhancement of the dileptons. In addition to the amplification that occurs whenever the system is inside the unstable region, as discussed above, there is an additional enhancement resulting from the parametric amplification caused by the approximately regular oscillation of the order parameter with a frequency near the $\sigma$ mass $[36,37]$.
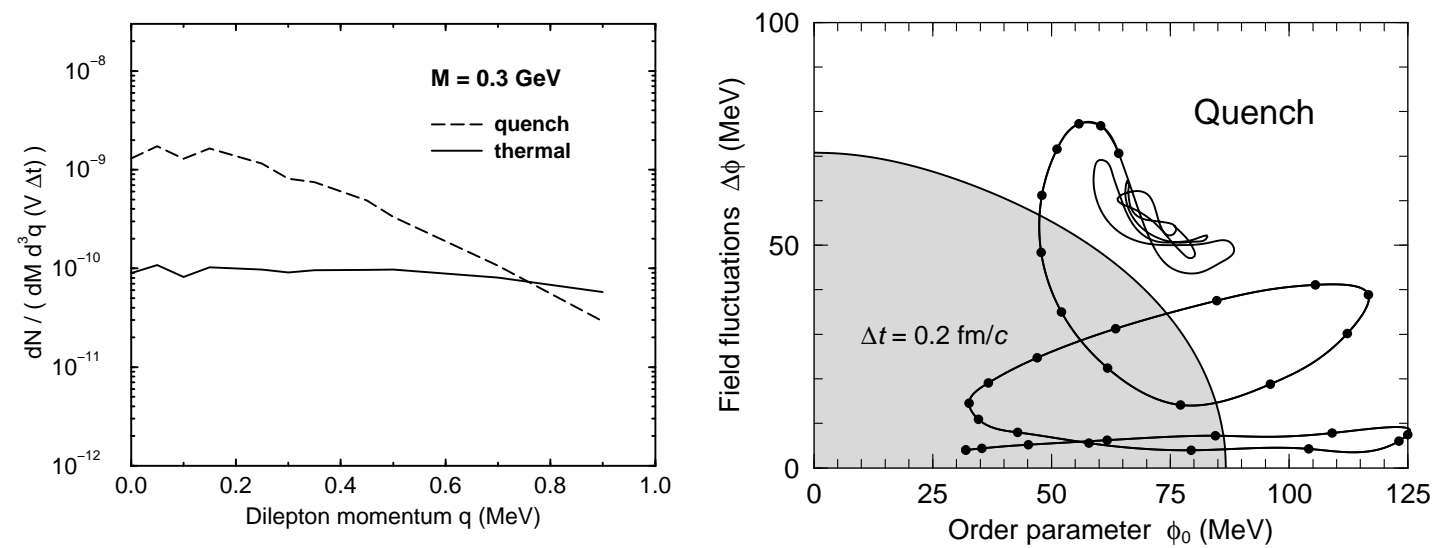

FIGURE 12. Left (12a): The dilepton production rate $d^{4} N /\left(d^{4} q V \Delta t\right)$ as a function of the magnitude of the dilepton momentum, for dilepton masses near $M=300 \mathrm{MeV}$ from either a thermal source at $T=140 \mathrm{MeV}$ or as obtained as a result of the quench depicted in the right panel [36]. Right (12b): The dynamical path after the same system has been quenched: (the field fluctuations have been nearly eliminated and the order parameter has been placed the supercirtical region with a value that ensures that the energy is the same as in the thermal source [38]. 


\section{Bjorken Rods}

We now turn to the study of a more refined scenario that exhibits some of the most important features expected in real collision events, namely rapid longitudinal expansion and finite transverse extension [13]. Specifically, we shall explore a socalled Bjorken rod: a rod-like geometry endowed with a longitudinal scaling expansion (not merely experiencing a Rayleigh cooling). Such systems have a finite (circular) extension in the transverse plane, and the local environment changes smoothly from hot longitudinally expanding matter in the bulk to vacuum outside.

Generally, the numerical propagation of fields exhibiting significant flow patterns, such as rapid expansion, is practically difficult due to the phase oscillations caused by the local boost. However, for idealized scaling expansions this complication can be eliminated by suitable variable transformations. For the longitudinal scaling expansion considered here, it is convenient to replace the usual fixed-frame spacetime variables $(x, y, z, t)$ with the comoving variables $(x, y, \eta, \tau)[24]$,

$$
t=\tau \cosh \eta, z=\tau \sinh \eta .
$$

Thus, $\tau=\left(t^{2}-z^{2}\right)^{1 / 2}$ is the proper time experienced in a system boosted along the $z$ axis with the local rapidity y equal to the value of $\eta=\frac{1}{2} \ln \left(\frac{t+z}{t-z}\right)$. The corresponding form of the field equation of motion is then obtained from the transformation of the d'Alembert operator,

$$
\square \equiv \partial_{t}^{2}-\partial_{x}^{2}-\partial_{y}^{2}-\partial_{z}^{2}=\frac{1}{\tau} \partial_{\tau} \tau \partial_{\tau}-\partial_{x}^{2}-\partial_{y}^{2}-\frac{1}{\tau^{2}} \partial_{\eta}^{2} .
$$

The equation of motion can be readily solved numerically by application of the leapfrog method, once the initial field $\boldsymbol{\phi}(\boldsymbol{r})$ and its time derivative $\boldsymbol{\psi}(\boldsymbol{r})$ are specified (see next page).

When analyzing the asymptotic field for the Bjorken rod, it is natural to perform a Fourier transformation in the transverse plane,

$$
\boldsymbol{\phi}_{\mathbf{k}}(\eta)=\int \frac{d^{2} \boldsymbol{\rho}}{\Omega_{\perp}} \boldsymbol{\phi}(\boldsymbol{\rho}, \eta) \mathrm{e}^{-i \mathbf{k} \cdot \boldsymbol{\rho}}, \boldsymbol{\psi}_{\mathbf{k}}(\eta)=\int \frac{d^{2} \boldsymbol{\rho}}{\Omega_{\perp}} \boldsymbol{\psi}(\boldsymbol{\rho}, \eta) \mathrm{e}^{-i \mathbf{k} \cdot \boldsymbol{\rho}},
$$

where $\Omega_{\perp}=L_{x} L_{y}$ denotes the transverse area of the spatial lattice. Furthermore, the position in the transverse plane is denoted by $\boldsymbol{\rho}=(x, y)$ and the transverse wave number is $\mathbf{k}=\left(k_{x}, k_{y}\right)$. For large times the longitudinal velocity of a given part of the system is given by $v_{z} \rightarrow z / t=\tanh (\eta)$. Thus, in that limit, the coordinate $\eta$ equals the rapidity, $\eta \rightarrow \mathrm{y}=\tanh ^{-1}\left(v_{z}\right)$.

At present, we are only interested in observables based on the pion component of the chiral field, which are expressed in terms of the spherical components, $\boldsymbol{\pi}=$ $\left(\pi_{-}, \pi_{0}, \pi_{+}\right)$. The mean number of pions (a given charge state $j$ ) emerging with transverse wave vector $\mathbf{k}$ and a rapidity $\mathrm{y}$ in the interval $\left(\mathrm{y}_{1}, \mathrm{y}_{2}\right)$ is then given by

$$
\bar{n}_{\mathbf{k}}^{(j)}\left(\mathrm{y}_{1}, \mathrm{y}_{2}\right)=\Omega_{\perp} \int_{\mathrm{y}_{1}}^{\mathrm{y}_{2}} d \eta\left|\sqrt{\frac{m_{k}}{2}} \boldsymbol{\phi}_{\mathbf{k}}(\eta)+\frac{i}{\sqrt{2 m_{k}}} \boldsymbol{\psi}_{\mathbf{k}}(\eta)\right|^{2} .
$$




\section{Preparation of the Rod}

In order to prepare the initial field configuration for the rod, we proceed at first in the same manner as for the preparation of the matter scenario addressed above and sample the field configuration $\left(\boldsymbol{\phi}_{\text {box }}, \boldsymbol{\psi}_{\text {box }}\right)$ from a thermal ensemble describing macroscopically uniform matter within the overall box containing the calculational lattice. This field configuration can be uniquely decomposed into its spatial average, the order parameter, and the remainder, the fluctuating part of the field,

$$
\phi_{\mathrm{box}}(\boldsymbol{\rho}, \eta)=\underline{\phi}+\delta \phi(\rho, \eta), \boldsymbol{\psi}_{\mathrm{box}}(\boldsymbol{\rho}, \eta)=\underline{\boldsymbol{\psi}}+\delta \boldsymbol{\psi}(\boldsymbol{\rho}, \eta)
$$

In order to obtain the field configuration describing the initial state of the rod, $\left(\phi_{\text {rod }}, \boldsymbol{\psi}_{\text {rod }}\right)$, we rescale the two parts based on a specified local temperature $T(\rho)$ and then recombine them into the desired initial conditions,

$$
\begin{aligned}
\boldsymbol{\phi}_{\mathrm{rod}}\left(\boldsymbol{\rho}, \eta, \tau_{0}\right) & =g(\rho)\left[\underline{\boldsymbol{\phi}}-\boldsymbol{\phi}_{\mathrm{gs}}\right]+\boldsymbol{\phi}_{\mathrm{gs}}+\boldsymbol{h}(\rho) \circ[\boldsymbol{\delta} \boldsymbol{\phi}(\boldsymbol{\rho}, \eta)-\underline{\boldsymbol{\phi}}]+\underline{\boldsymbol{\phi}} \\
\boldsymbol{\psi}_{\mathrm{rod}}\left(\boldsymbol{\rho}, \eta, \tau_{0}\right) & =g(\rho)\left[\underline{\boldsymbol{\psi}}-\boldsymbol{\psi}_{\mathrm{gs}}\right]+\boldsymbol{\psi}_{\mathrm{gs}}+\boldsymbol{h}(\rho) \circ[\boldsymbol{\delta} \boldsymbol{\psi}(\boldsymbol{\rho}, \eta)-\underline{\boldsymbol{\psi}}]+\underline{\boldsymbol{\psi}}
\end{aligned}
$$

where $\boldsymbol{\phi}_{\mathrm{gs}}=\left(f_{\pi}, \mathbf{0}\right)$ and $\boldsymbol{\psi}_{\mathrm{gs}}=(0, \mathbf{0})$ are the vacuum values. The scaling coefficients for the order parameter and the fluctuations, $g(\rho)$ and $\boldsymbol{h}(\rho)$, are obtained by using the corresponding equilibrium values for the specified local temperature $T(\rho)$,

$$
g(\rho)=\frac{\phi_{0}(T(\rho))-f_{\pi}}{\phi_{0}\left(T_{0}\right)-f_{\pi}}, \quad h_{\sigma}(\rho)=\left(\frac{\delta \sigma_{T}^{2}}{\delta \sigma_{T_{0}}^{2}}\right)^{\frac{1}{2}}, \quad h_{\pi}(\rho)=\left(\frac{\delta \pi_{T}^{2}}{\delta \pi_{T_{0}}^{2}}\right)^{\frac{1}{2}}
$$

By proceeding in this manner we ensure that the local environment, as characterized by the order parameter and the field fluctuations, reflects approximately thermal equilibrium in matter held at the local temperature $T$, which decreases from its bulk value $T_{0}$ to zero according to the prescribed radial profile $T(\rho)$.

The initial system is illustrated in Fig. 13 for $T_{0}=240 \mathrm{MeV}$. Since the local temperature drops steadily as a function of the transverse distance $\rho$, moving out along the abscissa corresponds to reducing the temperature (though not at a steady rate). As one moves out through the surface, the order parameter increases steadily from its reduced value $(\approx 27 \mathrm{MeV})$ in the hot bulk region towards its vacuum value $f_{\pi}(=92 \mathrm{MeV})$, and the local thermal fluctuations drop correspondingly towards zero. (Since generally $\mu_{\sigma}>\mu_{\pi}$, the fluctuations along a pion direction exceed those in the $\sigma$ direction.) The resulting profiles of the effective masses also reflect their temperature dependencies: Starting from nearly degenerate values $(\approx 300 \mathrm{MeV})$ in the hot interior, where chiral symmetry is approximately restored, $\mu_{\pi}$ and $\mu_{\sigma}$ diverge steadily towards their free values of $138 \mathrm{MeV}$ and $600 \mathrm{MeV}$, respectively. For higher values of the central temperature $T_{0}$, the central value of the order parameter is smaller and the effective masses are larger (and even closer in value) and $\mu_{\sigma}$ will in fact exhibit a dip in the surface region as the local temperature passes through the critical region. 
Initial transverse profile of Bjorken rod

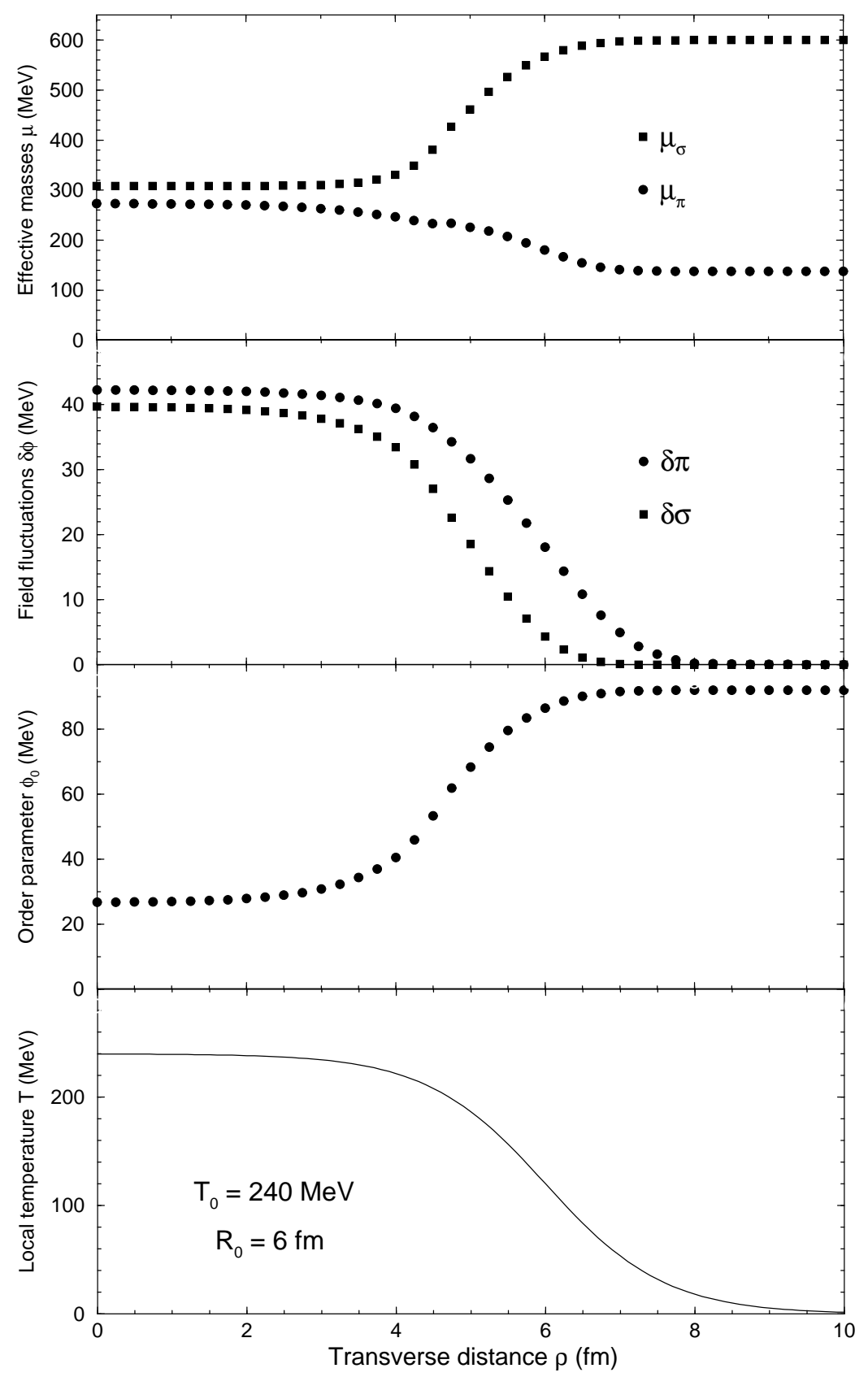

FIGURE 13. Initial transverse profiles of key quantities for a Bjorken rod prepared with a radius of $R_{0}=6 \mathrm{fm}$ and a central temperature of $T_{0}=240 \mathrm{MeV}$ : the specified local temperature $T(\rho)$, the order parameter $\phi_{0}(\rho)$, the mean fluctuation of the field in a given $\mathrm{O}(4)$ direction, $\delta \sigma(\rho)$ and $\delta \pi(\rho)$, and the corresponding effective quasiparticle masses, $\mu_{\sigma}(\rho)$ and $\mu_{\pi}(\rho)$ (from Ref [13]). 


\section{Rod Dynamics}

After preparing the field configuration of the rod, the dynamical propagation is readily obtained by solving the field equation (1) in the usual manner. Since the system is no longer macroscopically uniform, it is more complicated to discuss. but it is especially instructive to see how the bulk region develops. For this purpose, we compare with uniform matter prepared at the bulk temperature $T_{0}$ and endowed with a corresponding longitudinal expansion. Such a system may be denoted Bjorken matter and represents the environment that would be obtained in interior of a rod with a very large radius, $R_{0} \rightarrow \infty$.

The resulting evolution of the order parameter and the field fluctuations is illustrated in Fig. 14 for a rod with $T_{0}=240 \mathrm{MeV}$ and $R_{0}=6 \mathrm{fm}$. The corresponding quantities for Bjorken matter prepared with the same value of $T_{0}$ are also shown. It is seen that the environment in the interior of the rod evolves in a manner quantitatively very similar to that of the corresponding matter scenario throughout the first complete oscillation of the the order parameter, which takes about $4 \mathrm{fm} / c$. At around that point in time, the decompression wave has arrived from the rod surface and the self-generated transverse expansion now extends over the entire cross section of the rod. As a consequence, the relaxation progresses faster than it would in matter where this effect is absent.
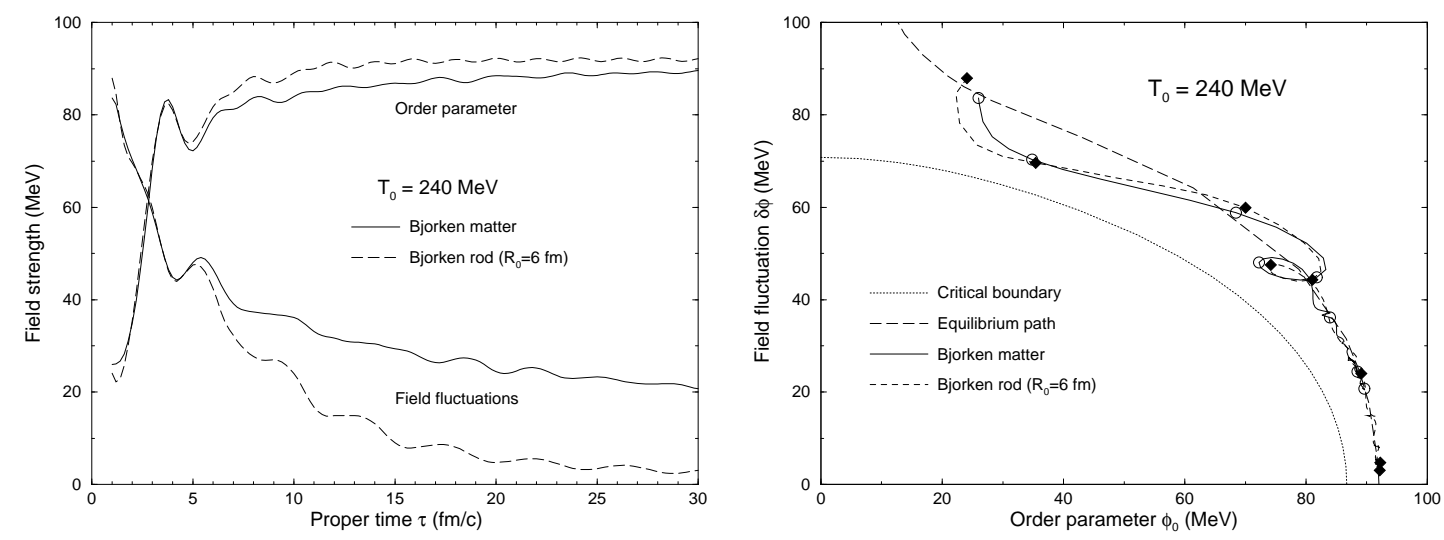

FIGURE 14. Left (14a): The time dependence of $\phi_{0}$, the magnitude of the order parameter (increasing curves), and $\delta \phi$, the associated dispersion of the field fluctuations (decreasing curves), in both Bjorken matter prepared with $T_{0}=240 \mathrm{MeV}$ (solid curves) and the corresponding Bjorken rod with $R_{0}=6 \mathrm{fm}$ (dashed curves). The information for the rod has been obtained by averaging over a hollow cylindrical volume, $1<\rho(\mathrm{fm})<3$. Right $(14 \mathrm{~b})$ : The corresponding phase evolution of the interior of the Bjorken rod (dashed path, filled diamonds), and the associated Bjorken matter (solid path, open circles). The equilibrium path is shown by the short-dashed curve while the dotted curve delineates the region of instability within which the field is supercritical. The symbols give the path locations at successive proper times $\tau(\mathrm{fm} / c)=1,2,3,4,5,10,20,30$. [13] 

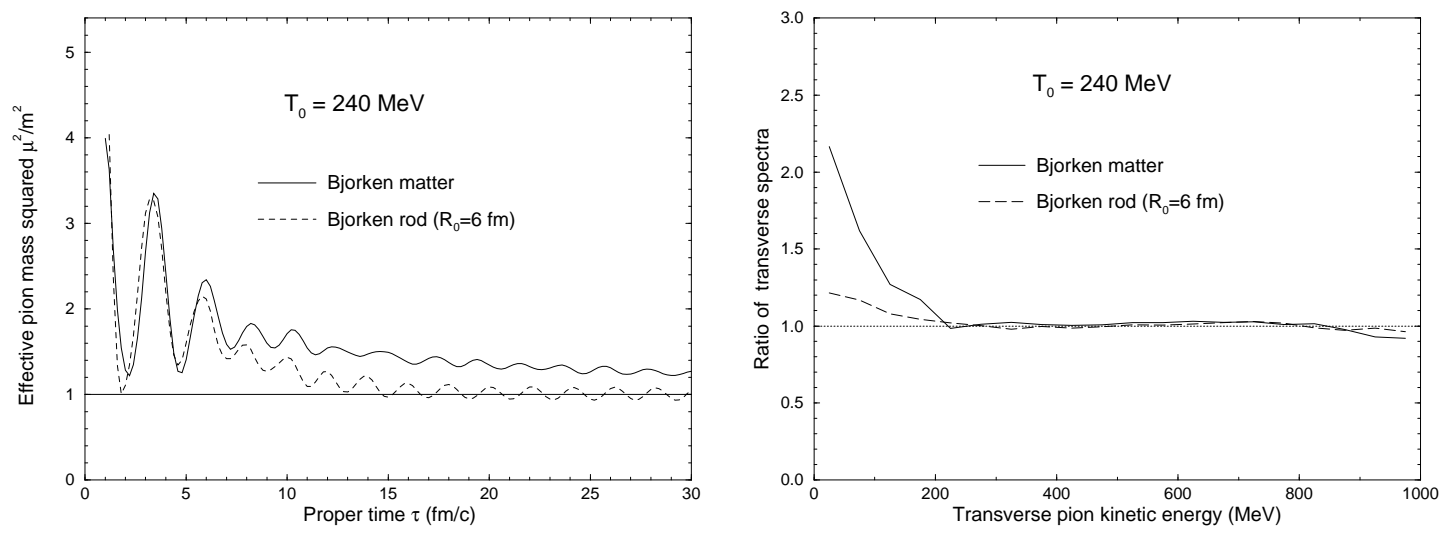

FIGURE 15. Left panel (15a): Time evolution of the square of the effective pion mass, $\mu_{\pi}^{2}(t)$ (divided by the square of its free mass $m_{\pi}$ ) for a Bjorken rod (dashed) prepared with $T_{0}=240 \mathrm{MeV}$ and $R_{0}=6 \mathrm{fm}$ as well as the corresponding result for Bjorken matter (solid). The information for the rod has been obtained by averaging over a hollow cylindrical volume, $1<\rho(\mathrm{fm})<3$. Right panel (15b): The ratio between the final transverse pion spectrum, $d^{3} N / d^{2} \boldsymbol{p}_{\perp} d \mathrm{y}$, and the associated equilibrium spectrum obtained by fitting the dynamical result with a Bose-Einstein form within the energy interval $200-1000 \mathrm{MeV}$, for the longitudinally expanding rod having an initial radius of $R_{0}=6 \mathrm{fm}$ and with an initial bulk temperature of $T_{0}=240 \mathrm{MeV}$ (dashed curve), as well as the corresponding result for Bjorken matter. (These illustrations are from [13].)

The quicker relaxation of the field for the rod is also reflected in the behavior of the effective pion mass, as illustrated in Fig. 15a for the same case. Again we see that through the first several time units the effective mass in the interior of the rod follows closely the evolution of the effective mass extracted for the corresponding matter scenario, but it then drops much faster towards the free value. Furthermore, the oscillations in the effective mass persist for quite a long time, thus making it possible to achieve a significant degree of parametric amplification.

\section{Observables}

We now consider a few specific observables that are practically accessible in the analysis of actual experimental data. First we consider the transverse spectra of the emerging pions, $E d^{3} N / d^{3} \boldsymbol{p}=d^{3} N / d^{2} \mathbf{k} d \mathbf{y}$. As it turns out, the transverse spectral shape is well approximated by an equilibrium form above kinetic energies of $200 \mathrm{MeV}$ or so and one may thus extract an spectral effective temperature. Moreover, it is instructive to divide by that overall shape. The resulting ratio between the dynamical spectrum and the corresponding Bose-Einstein form is displayed in Fig. 15b together with the corresponding matter result. Clearly, there is a significant enhancement of soft pions, although the effect is considerably smaller in the rod due to the presence of the surface. 

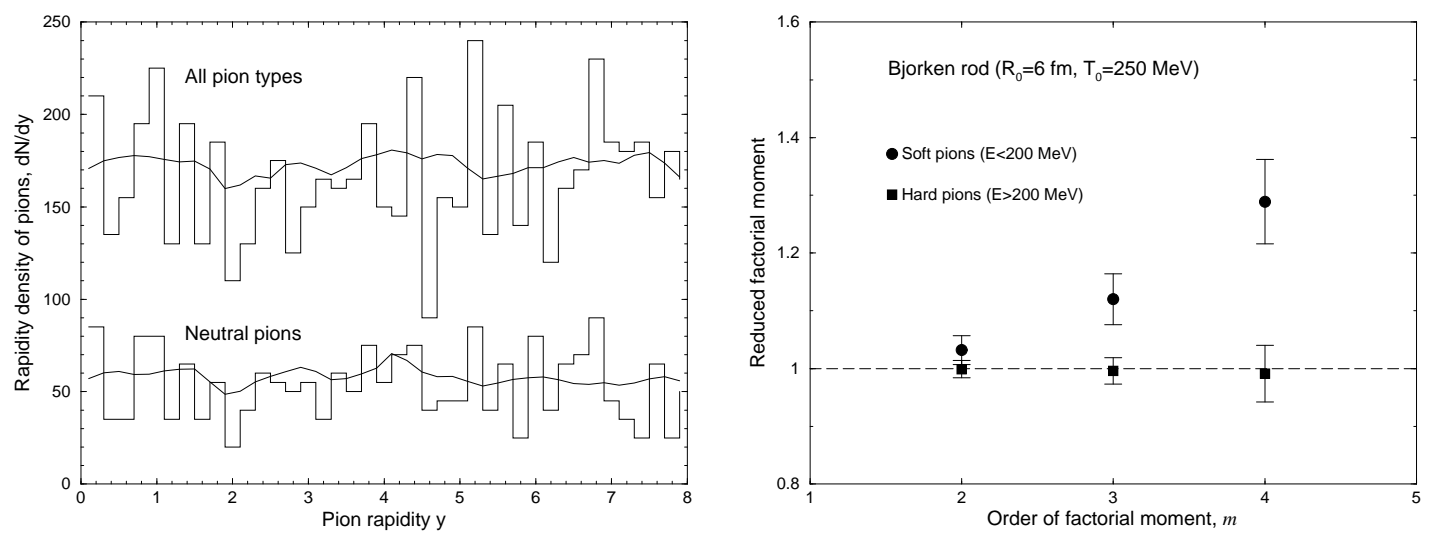

FIGURE 16. Left panel (16a): The rapidity density of pions for a single event prepared with $T_{0}=250 \mathrm{MeV}$ (with rapidity bins $\Delta \mathrm{y}=0.2$ ). The expected multiplicity based on the asymptotic field is indicated by the smooth solid curve, while the histogram shows the actual number as picked from the corresponding Poisson distributions. The top curves include all three pion types, whereas the bottom curves show the neutras only. Right panel (16b): The corresponding reduced factorial moments $\mathcal{M}_{m} / \bar{N}^{m}$ as a function of the order $m$, for either soft or hard pions. [13]

By invoking the relation (39), it is straightforward to calculate the expected number of pions in a given rapidity interval and within a specified transverse energy bin. This result varies from event to event, since the field configurations vary at the microscopic level as a result of the fluctuations in the ensemble of initial states. Moreover, for a given value of the expected number $\bar{n}$, the actual number of particles emerging is a stochastic variable $n$, since the state described by a given field has no well-defined particle number. For an exploratory study it may suffice to employ a Poisson form, $P_{\bar{n}}(n)=\bar{n}^{n} \exp (-\bar{n}) / n$ !. Figure 16a shows both the expected and the actual multiplicity ( $\bar{n}$ and $n$, respectively), as obtained for a single event.

In order to investigate whether the resulting multiplicity distributions contain deviations from Poisson statistics, it is useful to extract the factorial moments.

$$
\mathcal{M}_{m} \equiv \prec N(N-1) \cdots(N-m+1) \succ,
$$

where $N$ is the number of pions emitted in a given rapidity interval and the average is over all such intervals and events. They are shown in Fig. 16b. While the hard pions appear to be perfectly consistent with pure Poisson statistics, the soft pions exhibit a significant non-poissonian behavior. The character of the deviation of the soft factorial moments suggests that the source occasionally emits anomalously many pions, as one would expect if some modes are especially amplified. This feature is consistent with the enhancement of the soft spectrum and it can also be explored by other means of analyzing the data for anomalous fluctuations. Furthermore, standard event generators (UrQMD and HIJING) do not produce any such difference between soft and hard multiplicity fluctuations [39]. 


\section{CONCLUDING REMARKS}

In this brief lecture series, we have tried to give an impression of current efforts towards exploiting high-energy nuclear collisions to elucidate our understanding of chiral symmetry in strongly interacting systems. We have especially discussed certain key aspects of the treatment (while others had to be left out), hoping thereby to make the rapidly growing literature on the subject more accessible. We have also made contact with experiment by illustrating how certain observables may carry signals of the expected non-equilibrium DCC dynamics.

Our discussion has been carried out within the framework of the linear $\sigma$ model which was treated in a semi-classical manner. Comparisons with more sophisticated approaches (such as that of Ref. [41]) as well as dynamical self-consistency tests [15] have suggested that this simple treatment is in fact semi-quantitatively reasonable. Nevertheless, refinements would be desirable in a number of respects.

One obvious challenge is to go beyond the semi-classical level and invoke actual quantum field theory. The practical treatment of real-time quantum field theory in an environment that is neither uniform nor thermal presents an interesting but formidable challenge. Fortunately, it appears possible to incorporate the quantitatively most important effects into the numerical DCC simulations, as is briefly illustrated in Appendix A. The principal lesson is that the ever present vacuum fluctuations act on an equal footing with the statistical fluctuations as seeds for dynamical amplification and, in this regard, they appear to be at least as important.

Another direction of desirable improvement concerns the incorporation af additional degrees of freedom, since the limitation to the $(\sigma, \boldsymbol{\pi})$ space is a drastic simplification, especially at the high energy densities occuring at the early stage. Particularly important is the inclusion of strangeness, as is briefly illustrated in Appendix B. This extension leads to significant modifications of the results but it also enriches the dynamics and offers additional possible signals.

Thus, many theoretical challenges remain, with regard to both the formal refinement of the treatment and the implementation of the theory into a practical tool that can provide specific guidance for the design and analysis of the experiments. With the recently completed RHIC facility now beginning to yield data, the need for further advances has intensified.

This work was supported by the Director, Office of Energy Research, Office of High Energy and Nuclear Physics, Nuclear Physics Division of the U.S. Department of Energy under Contract No. DE-AC03-76SF00098. 


\section{A: QUANTUM FIELD EFFECTS}

A full quantum-field treatment of the chiral dynamics is beyond current reach and the dynamical simulation studies have therefore employed classical fields. Although much valuable insight can been gained in this manner, it is important to recognize that such treatments are not always quantitatively accurate. This is perhaps best illustrated by considering a free pionic mode $\mathbf{k}$ with a time-dependent frequency, $\omega_{k}^{2}(t)=k^{2}+\mu^{2}(t)$, where $\mu^{2}(t)$ has a given form. The equation of motion for the corresponding field operator is $\left[\square+\omega_{k}^{2}(t)\right] \hat{\boldsymbol{\phi}}_{\mathbf{k}}(t)=0$, and the associated timeevolution operator can be determined (see Ref. [40]).

If the initial occupancy of the mode is $n_{\mathbf{k}}^{\text {init }}$, then the final occupancy becomes

$$
n_{\mathbf{k}}^{\text {final }}=X_{k}\left[n_{\mathbf{k}}^{\text {init }}+\frac{1}{2}\right]-\frac{1}{2}>X_{k} n_{\mathbf{k}}^{\text {init }},
$$

after averaging over the initial state phase. This exceeds the classical result $X_{k} n_{\mathbf{k}}^{\text {init }}$, since the amplification coefficient is generally larger than unity, $X_{k} \geq 1$. The above expression brings out the fact that the quantum fluctuations and the statistical fluctuations combine in a democratic fashion as seeds for the parametric amplification. A quantitative impression of their relative importance can be gained from Fig. 17a. It is evident that the thermal occupancies are never large compared to one half. Consequently, the vacuum fluctuations are never negligible.

Efforts to develop a suitable quantum-field treatment are underway [42] and an illustration is given in Fig. 17b. It is seen that pions with momenta around $200 \mathrm{MeV} / c$ are produced, even if the initial state contained no quasiparticles at all (the initial presence of thermal excitations will then increase the yield further). This spectral shape reflects the regular temporal modulation of the pion mass.
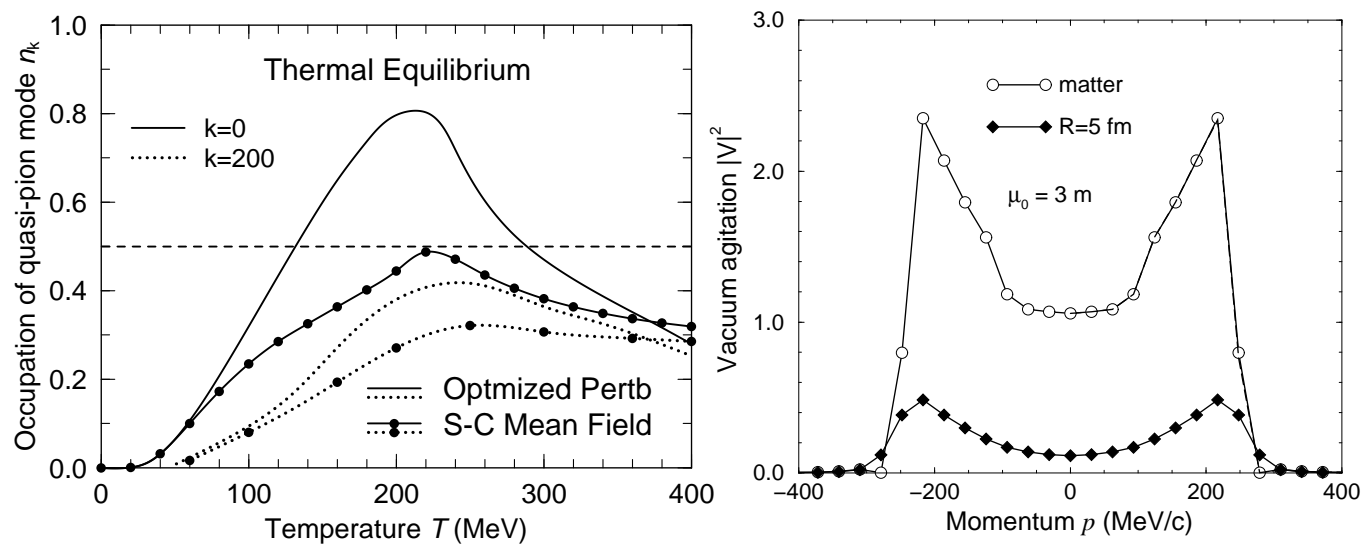

FIGURE 17. Left panel (17a): The temperature dependence of the thermal occupanices of the quasipions as obtained with either a semi-classicial treatment [15] or an optimized perturbation method [41]. Right panel (17b): The final pion spectrum arising solely from amplification of the vacuum fluctuations in one-dimensional systems with given effective mass functions $\mu_{\pi}^{2}(x, t)$ of forms similar to those obtained for Bjorken rods (see fig. 15a) [42]. 


\section{B: INCLUSION OF STRANGENESS}

Since the temperatures of interest easily exceed the mass of the $s$ quark, it is expected that the strange degrees of freedom are agitated as well and thus strangeness should be incorporated into the description. The extension from $u d$ to $u d s$ enlarges the meson group from $\mathrm{SU}(2)$ to $\mathrm{SU}(3)$, which contains a total of 18 fields,

$$
\mathrm{SU}(3)[u d s]:\left\{\begin{array}{lllllllll}
\sigma, & a_{0}^{0}, & \zeta ; & a_{0}^{-}, & a_{0}^{+}, & K^{*-}, & K^{*+}, & K^{* 0}, & \bar{K}^{* 0} \\
\pi^{0}, & \eta_{8}, & \eta_{0} ; & \pi^{-}, & \pi^{+}, & K^{-}, & K^{+}, & K^{0}, & \bar{K}^{0}
\end{array} .\right.
$$

In equilibrium, both the $\sigma$ and the $\zeta$ fields have finite values, and so the order parameter acquires a strange component, $\sigma \rightarrow(\sigma, \zeta)$, where $\zeta=\langle\bar{s} s\rangle$. It is possible to apply the semi-classical treatment to this more complicated case as well [43].

As is evident from Fig. 18 (left), the inclusion of strangeness severely impedes the restoration of chiral symmetry as the temperature is raised, thus casting doubt on the standard scenario in which approximate restoration is assumed to occur once $T$ exceeds a few hundred $\mathrm{MeV}$.

On the other hand, as illustrated in Fig. 18 (right), the dynamics of the order parameter becomes more intricate. As a result, the kaon modes may experience parametric amplification in analogy to what happens for the pions. For an idealized source, the neutral pion fraction $f_{\pi}=\pi^{0} /\left(\pi^{+}+\pi^{-}+\pi^{0}\right)$ retains its $\mathrm{SU}(2)$ distribution, $P_{\pi}\left(f_{\pi}\right)=1 / 2 \sqrt{f_{\pi}}$, and the neutral kaon fraction $f_{K}=\left(K^{0}+\bar{K}^{0}\right) /\left(K^{+}+K^{-}+K^{0}+\bar{K}^{0}\right)$ has a uniform distribution, $P_{K}\left(f_{K}\right)=1$.
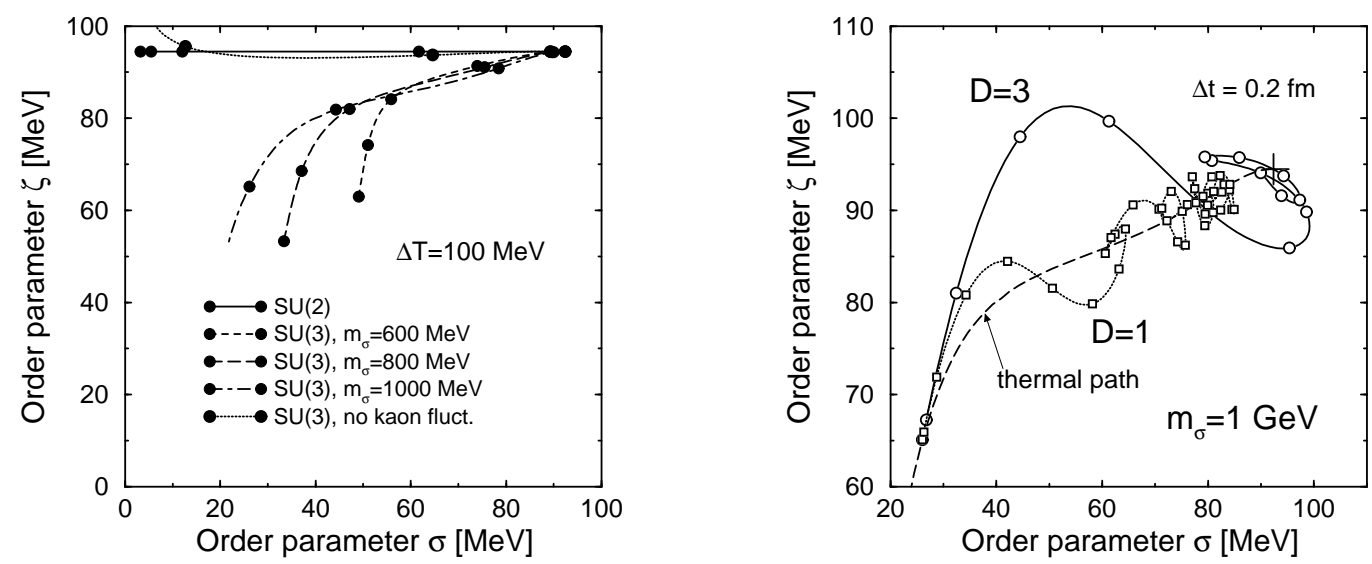

FIGURE 18. Left panel (18a): The thermal path of the two order parameter $(\sigma, \zeta)$ for various cases of interest, as indicated. The solid dots are plotted in temperature steps of $100 \mathrm{MeV}$. All paths start from the vacuum point at the upper-right corner and the points for $T=100 \mathrm{MeV}$ are still very close to the vacuum point. Right panel (18b): The dynamical path of the order parameter $(\sigma, \zeta)$ as a result of a pseudoexpansion in one and three dimensions, starting from equilibrium at $T=400 \mathrm{MeV}$. Equidistant time steps with $\Delta \tau=0.2 \mathrm{fm} / c$ are indicated. The thermal path is shown by the long-dashed curve, while the cross indicates the vacuum point, towards which all trajectories converge in time. (These illustrations are from Ref. [43].) 


\section{REFERENCES}

1. K. Rajagopal, in Quark-Gluon Plasma 2, Ed. R. Hwa, World Scientific (1995).

2. J.-P. Blaizot and A. Krzywicki, Acta Phys. Polon. B27, 1687 (1996).

3. J.D. Bjorken, Acta Phys. Polon. B28, 2773 (1997).

4. V. Koch, J. Mod. Phys. E6, 203 (1997).

5. M. Gell-Mann and M. Levy, Nuov. Cim. 16, 705 (1960).

6. K. Rajagopal, F. Wilczek, Nucl. Phys. B404, 577 (1993).

7. M. Asakawa, Z. Huang, and X.-N. Wang, Phys. Rev. Lett. 74, 3126 (1995).

8. L.P. Csernai and I.N. Mishustin, Phys. Rev. Lett. 74, 5005 (1995).

9. J. Randrup, Phys. Rev. Lett. 77, 1226 (1996).

10. G. Amelino-Camelia, J.D. Bjorken and S.E. Larsson, Phys. Rev. D56, 6942 (1997).

11. A. Abada and M.C. Birse, Phys. Rev. D57, 292 (1998).

12. D. Kaiser, Phys. Rev. D59, 117901 (1999).

13. T.C. Petersen and J. Randrup, Phys. Rev. C61, 024906 (2000).

14. Z. Xu and C. Greiner, Phys. Rev. D62, 036012 (2000).

15. J. Randrup, Phys. Rev. D55, 1188 (1997).

16. G. Baym and G. Grinstein, Phys. Rev. D15, 2897 (1977).

17. J. Goldstone, Nuov. Cim. 19, 154 (1961).

18. L.D. Landau and E.M. Lifshitz, Statistical Physics, Pergamon, New York (1980).

19. Z. Huang and X.-N. Wang, Phys. Rev. D49, 4335 (1994).

20. S. Gavin and B. Müller, Phys. Lett. B329, 486 (1994).

21. D. Boyanovsky and H.J. de Vega, Phys. Rev. D51, 734 (1995).

22. F. Cooper, Y. Kluger, E. Mottola, and J.P. Paz, Phys. Rev. D51, 2377 (1995).

23. Y. Kluger, F. Cooper, E. Mottola, J. Paz, A. Kovner, Nucl. Phys. A590, 581c (1995).

24. J.D. Bjorken, Phys. Rev. D27, 140 (1983).

25. M.A. Lampert, J.F. Dawson, and F. Cooper, Phys. Rev. D54, 2213 (1996).

26. J. Randrup, Nucl. Phys. A616, 531 (1997).

27. A.A. Anselm, Phys. Lett. B217, 169 (1989).

28. A.A. Anselm and M.G. Ryskin, Phys. Lett. B266, 482 (1991).

29. J.D. Bjorken, K.L. Kowalski, C.C. Taylor, SLAC-PUB-6109; hep-ph/9309235 (1993).

30. K. Rajagopal and F. Wilczek, Nucl. Phys. B399, 395 (1993).

31. J.P. Blaizot and A. Krzywicki, Phys. Rev. D46, 246 (1992).

32. Z. Huang, I. Sarcevic. R. Thews, and X.N. Wang, Phys. Rev. D54, 750 (1996).

33. K. Rajagopal, Hirschegg Winter Workshop XXV; hep-ph/9703258 (1997).

34. J. Randrup and R.L. Thews, Phys. Rev. D56, 4392 (1997).

35. Z. Huang and X.N. Wang, Phys. Lett. B383, 457 (1996).

36. Y. Kluger, V. Koch, J. Randrup, and X.N. Wang, Phys. Rev. C57, 280 (1998).

37. D. Boyanovsky, H.J. de Vega, R. Holman, S.P. Kumar, Phys. Rev. D56, 5233 (1997).

38. J. Randrup, Nucl. Phys. A630, 468c (1998).

39. M. Bleicher, J. Randrup, R. Snellings, X.-N. Wang, Phys. Rev. C62, 041901 (2000).

40. J. Randrup, Heavy Ion Phys. 9 (1999) 289.

41. S. Chiku and T. Hatsuda, Phys. Rev. D57, R6 (1998).

42. J. Randrup, Phys. Rev. C62, 064905 (2000).

43. J. Schaffner-Bielich and J. Randrup, Phys. Rev. C59, 3329 (1999). 